\title{
Optimization of the Two Fishermen's Profits Exploiting Three Competing Species Where Prices Depend on Harvest
}

\author{
Imane Agmour, Meriem Bentounsi, Naceur Achtaich, and Youssef El Foutayeni \\ Analysis, Modeling and Simulation Laboratory, Hassan II University, Casablanca, Morocco \\ Correspondence should be addressed to Youssef El Foutayeni; foutayeni@gmail.com
}

Received 21 August 2017; Accepted 13 November 2017; Published 26 December 2017

Academic Editor: Abid A. Lashari

Copyright ( 2017 Imane Agmour et al. This is an open access article distributed under the Creative Commons Attribution License, which permits unrestricted use, distribution, and reproduction in any medium, provided the original work is properly cited.

\begin{abstract}
Bioeconomic modeling of the exploitation of biological resources such as fisheries has gained importance in recent years. In this work we propose to define and study a bioeconomic equilibrium model for two fishermen who catch three species taking into consideration the fact that the prices of fish populations vary according to the quantity harvested; these species compete with each other for space or food; the natural growth of each species is modeled using a logistic law. The main purpose of this work is to define the fishing effort that maximizes the profit of each fisherman, but all of them have to respect two constraints: the first one is the sustainable management of the resources and the second one is the preservation of the biodiversity. The existence of the steady states and their stability are studied using eigenvalue analysis. The problem of determining the equilibrium point that maximizes the profit of each fisherman leads to Nash equilibrium problem; to solve this problem we transform it into a linear complementarity problem (LCP); then we prove that the obtained problem (LCP) admits a unique solution that represents the Nash equilibrium point of our problem. We close our paper with some numerical simulations.
\end{abstract}

\section{Introduction}

Overfishing leads to resource destruction, that is why there is an increasing need for the bioeconomic modeling tool that evaluates the biological and economic effects of different harvesting strategies directed at extracting the long-term maximum sustainable production while avoiding the risk of recruitment overfishing. The techniques and issues associated with the bioeconomic modeling for the exploitation of marine resources have been discussed in detail by Clark and Munro [1, 2]. Clark and Munro [1] demonstrated that, with the aid of optimal control theory, fisheries economics can without difficulty be cast in a capital-theoretic framework yielding results that are both general and readily comprehensible. Chaudhuri [3] discussed the problem of combined harvesting of two competing fish species, each of which obeys the law of logistic growth; it is shown that the openaccess fishery may possess a bioeconomic equilibrium which drives one species to extinction. In this context, Chaudhuri [4] considered the problem of dynamic optimization of the exploitation policy connected with the combined harvesting of two competing fish species, each of which obeys the logistic growth law. Models on the combined harvesting of a two-species prey-predator fishery have been discussed by Chaudhuri and Ray [5]. Kar and Chaudhuri [6] studied the problem of harvesting two competing species in the presence of a predator species which feeds on both the competing species; a combined harvesting effort is devoted to the exploitation of the first two (prey) species while the third (predator) species is not harvested. Mchich et al. [7] proposed a specific stock-effort dynamic model; the stock corresponds to two fish populations growing and moving between two fishing zones, on which they are harvested by two different fleets; the effort represents the number of fishing vessels of the two fleets which operate on the two fishing zones; the bioeconomic model is a set of four ordinary differential equations governing the stocks and the fishing efforts in the two fishing areas; fish migration, as well as vessels displacements, between the two zones is assumed to take place at a faster time scale than the variation of the stocks and the changes of fleets sizes, respectively; the vessels movements between the two fishing areas are assumed to be stock dependent, that is, the larger the stock density is in a zone, the more the vessels tend to remain in it. 
Many mathematical models have been developed to describe the dynamics of fisheries; we can refer, for example, to El Foutayeni et al. [8] who in their work have built a bioeconomic equilibrium model for several fishermen who catch two fish species; in this work, the authors have showed that the problem of determining the equilibrium point that maximizes the profit of each fisherman is solved by using linear complementarity problem. El Foutayeni et al. [9] have also defined a bioeconomic equilibrium model for " $n$ " fishermen who catch three species; these species compete with each other for space or food; the natural growth of each species is modeled using a logistic law; the objective of their work is to calculate the fishing effort that maximizes the profit of each fisherman at biological equilibrium by using the generalized Nash equilibrium problem.

Most bioeconomic models do not take into account the variational of the price of fish population. Usually, the existing models consider that the prices of the fish populations are constants. In this context, El Foutayeni and Khaladi [10, 11] have presented a bioeconomic model of fish populations taking into consideration the fact that the prices of fish populations vary according to the quantity harvested. But in these articles they assumed the existence of a single fisherman.

This paper is situated in this general context; in this work we present a bioeconomic model for three species which compete with each other for space or food and each of which obeys the law logistic growth. These species are caught by two fishermen. We will assume that the price of the fish population increases with decreasing harvest and the price of the fish population decreases with the increase of the harvest, but the minimum price is equal to a fixed positive constant. The aim of this paper consists in determining the fishing effort strategy adopted by each fisherman to maximize its income under two assumptions; the first one is the sustainable management of the resources, and the second one is the preservation of the biodiversity.

The paper is structured as follows. In Section 2, we give a description of the biological model of fish populations; we will define the mathematical model and study the stability of the equilibrium of our system. In Section 3, we give the bioeconomic model of the fish populations taking into consideration the fact that the prices of fish populations vary according to the quantity harvested; in this section we prove that the resolution of bioeconomic equilibrium model of the three fish populations is equivalent to solving a Nash equilibrium problem and then we show that the latter problem is equivalent to a linear complementarity problem, then we prove that the obtained problem (LCP) admits a unique solution that represents the Nash equilibrium of our problem. Some numerical simulations are given in Section 4 to illustrate the results. Finally, in Section 5 we give a conclusion.

\section{The Biological Model of Fish Populations}

The aim of this section is to define a biological model of three marine species that compete with each other for space or food and whose natural growth of each is obtained by means of a logistic law. We study the existence of the steady states and their stability using eigenvalue analysis and Routh-Hurwitz stability criterion.

2.1. The Mathematical Model and Hypotheses. The evolution of the biomass of the first species is given by the following mathematical equation:

$$
\begin{aligned}
\dot{x}_{1}(t)= & r_{1} x_{1}(t)\left(1-\frac{x_{1}(t)}{K_{1}}\right)-c_{12} x_{1}(t) x_{2}(t) \\
& -c_{13} x_{1}(t) x_{3}(t),
\end{aligned}
$$

where $x_{1}(t)$ is the biomass of population $1 ; r_{1}$ is the intrinsic growth rate of species $1 ; K_{1}$ is the carrying capacity for species $1 ; c_{12}$ is the coefficient of competition between species 2 and species 1 ; and $c_{13}$ is the coefficient of competition between species 3 and species 1 .

The evolution of the biomass of the second population is given by the following mathematical equation:

$$
\begin{aligned}
\dot{x}_{2}(t)= & r_{2} x_{2}(t)\left(1-\frac{x_{2}(t)}{K_{2}}\right)-c_{21} x_{1}(t) x_{2}(t) \\
& -c_{23} x_{2}(t) x_{3}(t),
\end{aligned}
$$

where $x_{2}(t)$ is the biomass of population $2 ; r_{2}$ is the intrinsic growth rate of species $2 ; K_{2}$ is the carrying capacity for species $2 ; c_{21}$ is the coefficient of competition between species 1 and species 2 ; and $c_{23}$ is the coefficient of competition between species 3 and species 2 .

The evolution of the biomass of the third species is given by the following mathematical equation:

$$
\begin{aligned}
\dot{x}_{3}(t)= & r_{3} x_{3}(t)\left(1-\frac{x_{3}(t)}{K_{3}}\right)-c_{31} x_{1}(t) x_{3}(t) \\
& -c_{32} x_{2}(t) x_{3}(t),
\end{aligned}
$$

where $x_{3}(t)$ is the biomass of population $3 ; r_{3}$ is the intrinsic growth rate $3 ; K_{3}$ is the carrying capacity for the species of species $3 ; c_{31}$ is the coefficient of competition between species 1 and species 3 ; and $c_{32}$ is the coefficient of competition between species 2 and species 3 .

It is interesting to note that to assure the existence of the three species and their stability we should assume that

$$
r_{i}>c_{i j} K_{j}, \quad \forall i, j=1,2,3, \text { with } i \neq j .
$$

The evolution of the biomass of fish populations is modeled by the following equations:

$$
\begin{aligned}
\dot{x}_{1}(t)= & r_{1} x_{1}(t)\left(1-\frac{x_{1}(t)}{K_{1}}\right)-c_{12} x_{1}(t) x_{2}(t) \\
& -c_{13} x_{1}(t) x_{3}(t), \\
\dot{x}_{2}(t)= & r_{2} x_{2}(t)\left(1-\frac{x_{2}(t)}{K_{2}}\right)-c_{21} x_{1}(t) x_{2}(t) \\
& -c_{23} x_{2}(t) x_{3}(t), \\
\dot{x}_{3}(t)= & r_{3} x_{3}(t)\left(1-\frac{x_{3}(t)}{K_{3}}\right)-c_{31} x_{1}(t) x_{3}(t) \\
& -c_{32} x_{2}(t) x_{3}(t) .
\end{aligned}
$$


Let $x(t)=\left(x_{1}(t), x_{2}(t), x_{3}(t)\right)$ be the solution of system (5). Then all the solutions of the system (5) are nonnegative. To demonstrate that, we must recall that by [12] the system of equation

$$
\dot{x}=f\left(x_{1}, x_{2}, \ldots, x_{n}\right) \quad \text { with } x(t=0)=x_{0}
$$

is a positive system if and only if

$$
\begin{aligned}
\dot{x}_{i}=f_{i}\left(x_{1} \geq 0, \ldots, x_{i}=0, \ldots, x_{n} \geq 0\right) \geq 0 & \\
& \forall i \in[1 \cdots n] .
\end{aligned}
$$

In our case, for $x_{1}=0, x_{2}, x_{3} \geq 0$, we have $d x_{1} / d t=0 \geq 0$. By the same, for $x_{2}=0, x_{1}, x_{3} \geq 0$, we have $d x_{2} / d t=0 \geq 0$. Also for $x_{3}=0, x_{1}, x_{2} \geq 0$, we have $d x_{3} / d t=0 \geq 0$. Therefore, all the solutions of system (5) are nonnegative.

Theorem 1. All the solutions of system (5) which start in $\mathbb{R}_{+}^{3}$ are uniformly bounded.

Proof. We define the function

$$
W=x_{1}+x_{2}+x_{3} .
$$

Therefore, the time derivative along a solution of (5) is

$$
\begin{aligned}
\frac{d W}{d t}= & r_{1} x_{1}\left(1-\frac{x_{1}}{K_{1}}\right)+r_{2} x_{2}\left(1-\frac{x_{2}}{K_{2}}\right) \\
& +r_{3} x_{3}\left(1-\frac{x_{3}}{K_{3}}\right)-c_{12} x_{1} x_{2}-c_{13} x_{1} x_{3} \\
& -c_{21} x_{1} x_{2}-c_{23} x_{2} x_{3}-c_{31} x_{1} x_{3}-c_{32} x_{2} x_{3} .
\end{aligned}
$$

For each $\vartheta>0$, we have

$$
\begin{aligned}
\frac{d W}{d t}+\vartheta W= & r_{1} x_{1}\left(1-\frac{x_{1}}{K_{1}}\right)+r_{2} x_{2}\left(1-\frac{x_{2}}{K_{2}}\right) \\
& +r_{3} x_{3}\left(1-\frac{x_{3}}{K_{3}}\right)-c_{12} x_{1} x_{2}-c_{13} x_{1} x_{3} \\
& -c_{21} x_{1} x_{2}-c_{23} x_{2} x_{3}-c_{31} x_{1} x_{3} \\
& -c_{32} x_{2} x_{3}+\vartheta x_{1}+9 x_{2}+\vartheta x_{3} \\
\leq & r_{1} x_{1}\left(1-\frac{x_{1}}{K_{1}}\right)+r_{2} x_{2}\left(1-\frac{x_{2}}{K_{2}}\right) \\
& +r_{3} x_{3}\left(1-\frac{x_{3}}{K_{3}}\right)+\vartheta x_{1}+\vartheta x_{2}+\vartheta x_{3} \\
= & x_{1}\left[r_{1}\left(1-\frac{x_{1}}{K_{1}}\right)+\vartheta\right] \\
& +x_{2}\left[r_{2}\left(1-\frac{x_{2}}{K_{2}}\right)+\vartheta\right] \\
& +x_{3}\left[r_{3}\left(1-\frac{x_{3}}{K_{3}}\right)+\vartheta\right]
\end{aligned}
$$

$$
\begin{gathered}
=x_{1}\left(r_{1}+9\right)-\frac{r_{1}}{K_{1}} x_{1}^{2}+x_{2}\left(r_{2}+\vartheta\right) \\
-\frac{r_{2}}{K_{2}} x_{2}^{2}+x_{3}\left(r_{3}+\vartheta\right)-\frac{r_{3}}{K_{3}} x_{3}^{2} .
\end{gathered}
$$

We can easily show that

$$
\begin{aligned}
& -\frac{r_{1}}{K_{1}} x_{1}^{2}+x_{1}\left(r_{1}+9\right)-\frac{K_{1}}{4 r_{1}}\left(r_{1}+9\right)^{2} \leq 0, \\
& -\frac{r_{2}}{K_{2}} x_{2}^{2}+x_{2}\left(r_{2}+9\right)-\frac{K_{2}}{4 r_{2}}\left(r_{2}+9\right)^{2} \leq 0, \\
& -\frac{r_{3}}{K_{3}} x_{3}^{2}+x_{3}\left(r_{3}+9\right)-\frac{K_{3}}{4 r_{3}}\left(r_{3}+\vartheta\right)^{2} \leq 0 .
\end{aligned}
$$

Then

$$
\begin{aligned}
& -\frac{r_{1}}{K_{1}} x_{1}^{2}+x_{1}\left(r_{1}+9\right) \leq \frac{K_{1}}{4 r_{1}}\left(r_{1}+\vartheta\right)^{2}, \\
& -\frac{r_{2}}{K_{2}} x_{2}^{2}+x_{2}\left(r_{2}+\vartheta\right) \leq \frac{K_{2}}{4 r_{2}}\left(r_{2}+\vartheta\right)^{2}, \\
& -\frac{r_{3}}{K_{3}} x_{3}^{2}+x_{3}\left(r_{3}+\vartheta\right) \leq \frac{K_{3}}{4 r_{3}}\left(r_{3}+\vartheta\right)^{2} .
\end{aligned}
$$

Therefore, we can deduce that

$$
\begin{aligned}
\frac{d W}{d t}+\vartheta W \leq & \frac{K_{1}}{4 r_{1}}\left(r_{1}+\vartheta\right)^{2}+\frac{K_{2}}{4 r_{2}}\left(r_{2}+\vartheta\right)^{2} \\
& +\frac{K_{3}}{4 r_{3}}\left(r_{3}+\vartheta\right)^{2} .
\end{aligned}
$$

So the right-hand side is positive; therefore it is bounded for all $\left(x_{1}, x_{2}, x_{3}\right) \in \mathbb{R}_{+}^{3}$. Therefore we find a $\theta>0$ with $d W / d t+$ $\vartheta W<\theta$. Using the theory of differential inequality [13], we obtain

$$
\begin{aligned}
0 & \leq W\left(x_{1}, x_{2}, x_{3}\right) \\
& \leq \frac{\theta}{\vartheta}+\left[W\left(x_{1}(0), x_{2}(0), x_{3}(0)\right)-\frac{\theta}{\vartheta}\right] e^{-9 t}
\end{aligned}
$$

which, upon letting $t \rightarrow \infty$, yields $0 \leq W \leq \theta / \vartheta$.

Then, we have

$$
B=\left\{\left(x_{1}, x_{2}, x_{3}\right) \in \mathbb{R}_{+}^{3}: W<\frac{\theta}{\vartheta}+\varepsilon \text {, for any } \varepsilon>0\right\},
$$

where $B$ is the region in which all the solutions of system of (5) that start in $\mathbb{R}_{+}^{3}$ are confined.

2.2. The Steady States of the System. The steady states of the system of (5) are obtained by solving the system of equations 


$$
\begin{aligned}
& r_{1} x_{1}\left(1-\frac{x_{1}}{K_{1}}\right)-c_{12} x_{1} x_{2}-c_{13} x_{1} x_{3}=0 \\
& r_{2} x_{2}\left(1-\frac{x_{2}}{K_{2}}\right)-c_{21} x_{1} x_{2}-c_{23} x_{2} x_{3}=0 \\
& r_{3} x_{3}\left(1-\frac{x_{3}}{K_{3}}\right)-c_{31} x_{1} x_{3}-c_{32} x_{2} x_{3}=0
\end{aligned}
$$

This system of equations has eight solutions

$$
\begin{gathered}
P_{1}(0,0,0), P_{2}\left(K_{1}, 0,0\right), P_{3}\left(0, K_{2}, 0\right), P_{4}\left(0,0, K_{3}\right), \\
P_{5}\left(x_{1}^{(5)}, x_{2}^{(5)}, 0\right) \text {, where } \\
x_{1}^{(5)}=K_{1} r_{2} \frac{r_{1}-c_{12} K_{2}}{r_{1} r_{2}-c_{12} c_{21} K_{2} K_{1}}, \\
x_{2}^{(5)}=K_{2} r_{1} \frac{r_{2}-c_{21} K_{1}}{r_{1} r_{2}-c_{12} c_{21} K_{2} K_{1}}
\end{gathered}
$$

$$
\begin{aligned}
x_{1}^{*} & =\frac{K_{1}\left(r_{1} r_{2} r_{3}-r_{1} c_{23} c_{32} K_{2} K_{3}+r_{3} c_{12} c_{23} K_{2} K_{3}-r_{2} r_{3} c_{12} K_{2}-r_{2} r_{3} c_{13} K_{3}+r_{2} c_{13} c_{32} K_{2} K_{3}\right)}{\Delta}, \\
x_{2}^{*} & =\frac{K_{2}\left(r_{1} r_{2} r_{3}-r_{2} c_{13} c_{31} K_{1} K_{3}+r_{1} c_{23} c_{31} K_{1} K_{3}-r_{1} r_{3} c_{21} K_{1}-r_{1} r_{3} c_{23} K_{3}+r_{3} c_{13} c_{21} K_{1} K_{3}\right)}{\Delta}, \\
x_{3}^{*} & =\frac{K_{3}\left(r_{1} r_{2} r_{3}-r_{3} c_{12} c_{21} K_{1} K_{2}-r_{1} r_{2} c_{31} K_{1}+r_{1} c_{21} c_{32} K_{1} K_{2}+r_{2} c_{12} c_{31} K_{1} K_{2}-r_{1} r_{2} c_{32} K_{2}\right)}{\Delta}, \\
\Delta & =r_{1} r_{2} r_{3}-r_{1} c_{23} c_{32} K_{2} K_{3}-r_{2} c_{13} c_{31} K_{1} K_{3}-r_{3} c_{12} c_{21} K_{1} K_{2}+c_{12} c_{23} c_{31} K_{1} K_{2} K_{3}+c_{13} c_{21} c_{32} K_{1} K_{2} K_{3} .
\end{aligned}
$$

The system of (16) has several solutions, but only one of them can give the coexistence of the biomass of the three species; this solution is the point $P_{8}\left(x_{1}^{*}, x_{2}^{*}, x_{3}^{*}\right)$.

2.3. The Stability of the Steady States. The variational matrix of system (5) is

$$
J=\left[\begin{array}{ccc}
J_{11} & -c_{12} x_{1} & -c_{13} x_{1} \\
-c_{21} x_{2} & J_{22} & -c_{23} x_{2} \\
-c_{31} x_{3} & -c_{32} x_{3} & J_{33}
\end{array}\right],
$$

where

$$
\begin{aligned}
& J_{11}=r_{1}\left(1-\frac{2}{K_{1}} x_{1}\right)-c_{12} x_{2}-c_{13} x_{3}, \\
& J_{22}=r_{2}\left(1-\frac{2}{K_{2}} x_{2}\right)-c_{21} x_{1}-c_{23} x_{3}, \\
& J_{33}=r_{3}\left(1-\frac{2}{K_{3}} x_{3}\right)-c_{31} x_{1}-c_{32} x_{2} .
\end{aligned}
$$

Proposition 2. The point $P_{1}(0,0,0)$ is unstable.
Proof. The variational matrix of system (5) at the steady state $P_{1}(0,0,0)$ is

$$
J_{1}=\left[\begin{array}{ccc}
r_{1} & 0 & 0 \\
0 & r_{2} & 0 \\
0 & 0 & r_{3}
\end{array}\right] .
$$

The eigenvalues of $J_{1}$ are

$$
\begin{aligned}
& \lambda_{1}=r_{1}>0, \\
& \lambda_{2}=r_{2}>0, \\
& \lambda_{3}=r_{3}>0,
\end{aligned}
$$

then, the point $P_{1}(0,0,0)$ is unstable.

Let $r_{1}=2, r_{2}=1, r_{3}=3, c_{12}=0.009, c_{21}=0.007, c_{13}=$ $0.008, c_{23}=0.001, c_{31}=0.002, c_{32}=0.001, K_{1}=70, K_{2}=$ $50, K_{3}=40$ in appropriate units. Figure 1 shows the dynamical behaviors and phase space trajectory of the three marine species against time, beginning with the initial values $x(0)=$ $0.01, y(0)=0.01, z(0)=0.01$. By Figure 1 we find that the steady state point $P_{1}$ is unstable, and more precisely this point tends to the point $P_{8}$.

Proposition 3. The point $P_{2}\left(K_{1}, 0,0\right)$ is unstable if the conditions of existence given by (4) hold; if not, it is stable. 

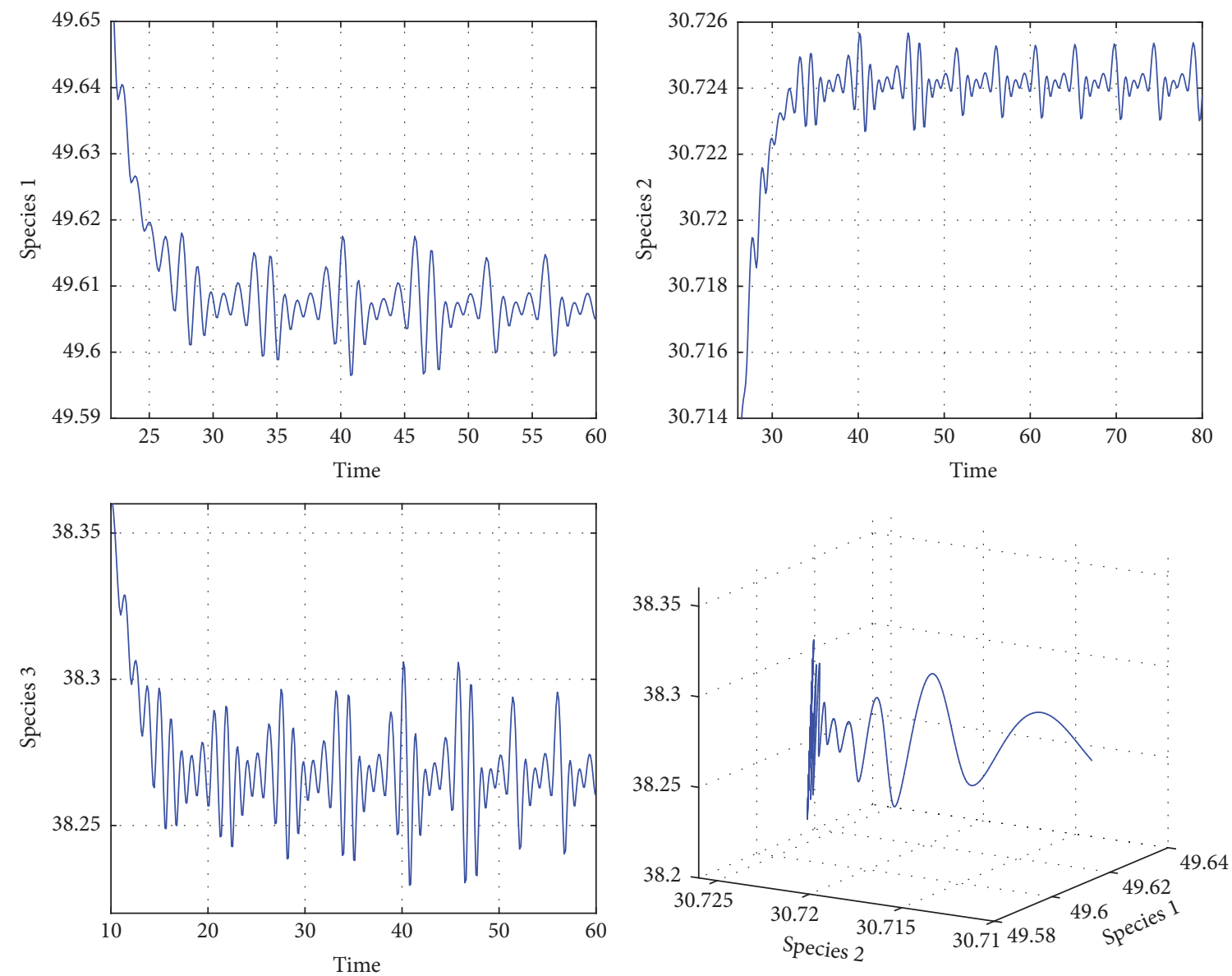

FIGURE 1: Dynamical behaviors and phase space trajectories of the three marine species.

Proof. The variational matrix of system (5) at the steady state $P_{2}\left(K_{1}, 0,0\right)$ is

$$
J_{2}=\left[\begin{array}{ccc}
-r_{1} & -c_{12} K_{1} & -c_{13} K_{1} \\
0 & r_{2}-c_{21} K_{1} & 0 \\
0 & 0 & r_{3}-c_{31} K_{1}
\end{array}\right]
$$

The eigenvalues of $J_{2}$ are

$$
\begin{aligned}
& \lambda_{1}=-r_{1}<0, \\
& \lambda_{2}=r_{2}-c_{21} K_{1}, \\
& \lambda_{3}=r_{3}-c_{31} K_{1}
\end{aligned}
$$

if

$$
\begin{aligned}
& r_{2}>c_{21} K_{1} \\
& r_{3}>c_{31} K_{1},
\end{aligned}
$$

then, the point $P_{2}\left(K_{1}, 0,0\right)$ is unstable; if not, it is stable.

Let $r_{1}=2, r_{2}=1, r_{3}=3, c_{12}=0.009, c_{21}=0.007, c_{13}=$ $0.008, c_{23}=0.001, c_{31}=0.002, c_{32}=0.001, K_{1}=70, K_{2}=$ $50, K_{3}=40$ in appropriate units. Figure 2 shows the dynamical behaviors and phase space trajectory of the three marine species against time, beginning with the initial values $x(0)=$
70, $y(0)=0.01, z(0)=0.01$. By Figure 2 we can see that the steady state point $P_{2}$ is unstable, and more precisely this point tends to the point $P_{8}$ too.

Proposition 4. The point $P_{3}\left(0, K_{2}, 0\right)$ is unstable if the conditions of existence given by (4) hold; if not, it is stable.

Proof. The variational matrix of system (5) at the steady state $P_{3}\left(0, K_{2}, 0\right)$ is

$$
J_{3}=\left[\begin{array}{ccc}
r_{1}-c_{12} K_{2} & 0 & 0 \\
-c_{21} K_{2} & -r_{2} & -c_{23} K_{2} \\
0 & 0 & r_{3}-c_{32} K_{2}
\end{array}\right] .
$$

The eigenvalues of $J_{3}$ are

$$
\begin{aligned}
& \lambda_{1}=r_{1}-c_{12} K_{2}, \\
& \lambda_{2}=-r_{2}<0, \\
& \lambda_{3}=r_{3}-c_{32} K_{2}
\end{aligned}
$$

if

$$
\begin{aligned}
& r_{1}>c_{12} K_{2}, \\
& r_{3}>c_{32} K_{2} ;
\end{aligned}
$$

therefore, the point $P_{3}\left(0, K_{2}, 0\right)$ is unstable; if not, it is stable. 

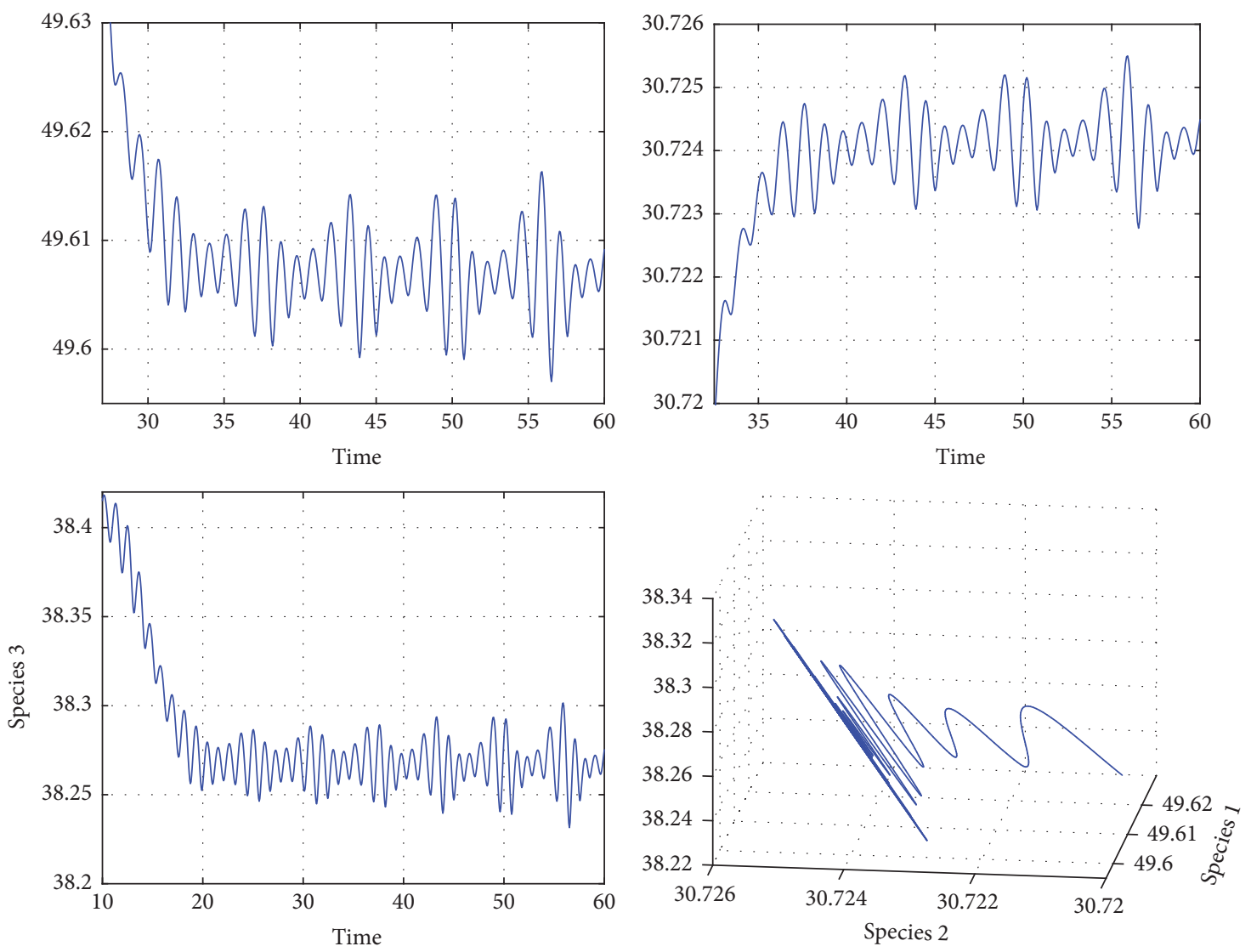

FIGURE 2: Dynamical behaviors and phase space trajectories of the three marine species.

Let $r_{1}=2, r_{2}=1, r_{3}=3, c_{12}=0.009, c_{21}=0.007, c_{13}=$ $0.008, c_{23}=0.001, c_{31}=0.002, c_{32}=0.001, K_{1}=70, K_{2}=$ $50, K_{3}=40$ in appropriate units. Figure 3 shows the dynamical behaviors and phase space trajectory of the three marine species against time, beginning with the initial values $x(0)=$ $0.01, y(0)=50, z(0)=0.01$. By Figure 3 we can see that the steady state point $P_{3}$ is also unstable and tends to the point $P_{8}$.

Proposition 5. The point $P_{4}\left(0,0, K_{3}\right)$ is unstable if the conditions of existence given by (4) hold; if not, it is stable.

Proof. The variational matrix of system (5) at the steady state $P_{4}\left(0,0, K_{3}\right)$ is

$$
J_{4}=\left[\begin{array}{ccc}
r_{1}-c_{13} K_{3} & 0 & 0 \\
0 & r_{2}-c_{23} K_{3} & 0 \\
-c_{13} K_{3} & -c_{32} K_{3} & -r_{3}
\end{array}\right]
$$

The eigenvalues of $J_{4}$ are

$$
\begin{aligned}
& \lambda_{1}=r_{1}-c_{13} K_{3}, \\
& \lambda_{2}=r_{2}-c_{23} K_{3}, \\
& \lambda_{3}=-r_{3}<0
\end{aligned}
$$

if

$$
\begin{aligned}
& r_{1}>c_{13} K_{3}, \\
& r_{2}>c_{23} K_{3},
\end{aligned}
$$

then, the point $P_{4}\left(0,0, K_{3}\right)$ is unstable; if not, it is stable.

Let $r_{1}=2, r_{2}=1, r_{3}=3, c_{12}=0.009, c_{21}=0.007, c_{13}=$ $0.008, c_{23}=0.001, c_{31}=0.002, c_{32}=0.001, K_{1}=70, K_{2}=$ $50, K_{3}=40$ in appropriate units. Figure 4 indicates the dynamical behaviors and phase space trajectory of the three marine species against time, beginning with the initial values $x(0)=0.01, y(0)=0.01, z(0)=40$. Following Figure 4 we can see that the steady state point $P_{3}$ is unstable and also tends to the point $P_{8}$.

Proposition 6. The point $P_{5}\left(x_{1}^{(5)}, x_{2}^{(5)}, 0\right)$ is unstable.

Proof. The variational matrix of system (5) at the steady state $P_{5}\left(x_{1}^{(5)}, x_{2}^{(5)}, 0\right)$ is

$$
J_{5}=\left[\begin{array}{ccc}
-\frac{r_{1}}{K_{1}} x_{1}^{(5)} & -c_{12} x_{1}^{(5)} & -c_{13} x_{1}^{(5)} \\
-c_{21} x_{2}^{(5)} & -\frac{r_{2}}{K_{2}} x_{2}^{(5)} & -c_{23} x_{2}^{(5)} \\
0 & 0 & r_{3}-c_{31} x_{1}^{(5)}-c_{32} x_{2}^{(5)}
\end{array}\right]
$$



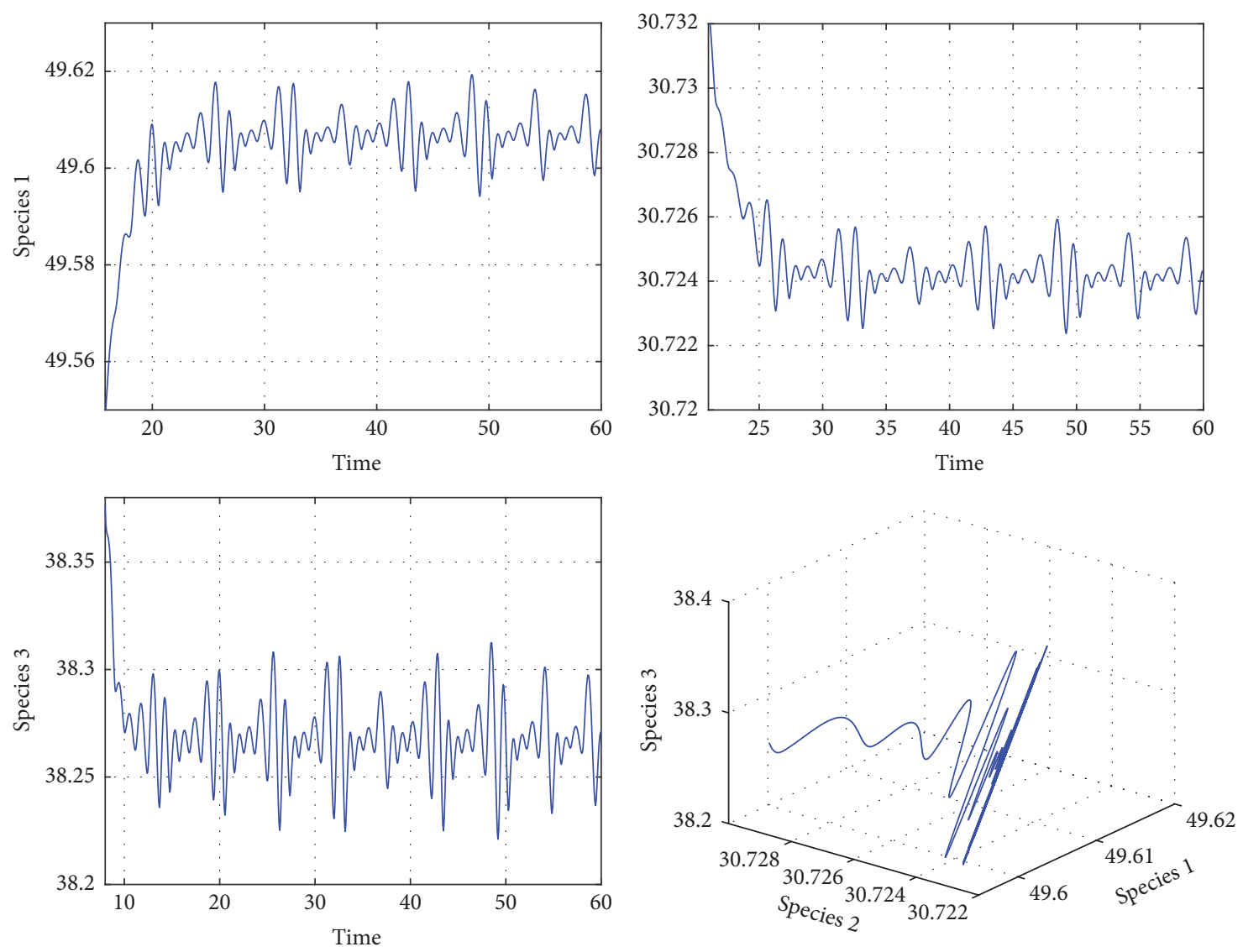

FIGURE 3: Dynamical behaviors and phase space trajectories of the three marine species.

The eigenvalues of $J_{5}$ are

$$
\begin{aligned}
& \lambda_{1}=-\frac{1}{2 K_{1} K_{2}}(M-\sqrt{N}), \\
& \lambda_{2}=-\frac{1}{2 K_{1} K_{2}}(M+\sqrt{N}), \\
& \lambda_{3}=r_{3}-c_{31} x_{1}^{(5)}-c_{32} x_{2}^{(5)},
\end{aligned}
$$

where

$$
\begin{aligned}
& M=r_{1} x_{1}^{(5)} K_{2}+K_{1} r_{2} x_{2}^{(5)}, \\
& N=\left[r_{1} x_{1}^{(5)} K_{2}-K_{1} r_{2} x_{2}^{(5)}\right]^{2}+4 K_{1}^{2} K_{2}^{2} c_{21} x_{2}^{(5)} c_{12} x_{1}^{(5)} .
\end{aligned}
$$

If

$$
\begin{aligned}
& r_{1}>c_{12} K_{2}, \\
& r_{2}>c_{21} K_{1}
\end{aligned}
$$

then, $\lambda_{3}>0$; if not, then $\lambda_{1}>0$. Therefore, the point $P_{5}\left(x_{1}^{(5)}\right.$, $\left.x_{2}^{(5)}, 0\right)$ is unstable in all cases.

Let $r_{1}=2, r_{2}=1, r_{3}=3, c_{12}=0.009, c_{21}=0.007, c_{13}=$ $0.008, c_{23}=0.001, c_{31}=0.002, c_{32}=0.001, K_{1}=70, K_{2}=$ $50, K_{3}=40$ in appropriate units. Figure 5 represents the dynamical behaviors and phase space trajectory of the three marine species against time, beginning with the initial values $x(0)=60, y(0)=28, z(0)=0.01$. Following Figure 5 we can deduce that the steady state point $P_{5}$ is unstable and also tends to the point $P_{8}$.

Proposition 7. The point $P_{6}\left(x_{1}^{(6)}, 0, x_{3}^{(6)}\right)$ is unstable.

Proof. The variational matrix of system (5) at the steady state $P_{6}\left(x_{1}^{(6)}, 0, x_{3}^{(6)}\right)$ is

$$
J_{6}=\left[\begin{array}{ccc}
-\frac{r_{1}}{k_{1}} x_{1}^{(6)} & -c_{12} x_{1}^{(6)} & -c_{13} x_{1}^{(6)} \\
0 & r_{2}-c_{21} x_{1}^{(6)}-c_{23} x_{3}^{(6)} & 0 \\
-c_{31} x_{3}^{(6)} & -c_{32} x_{3}^{(6)} & -\frac{r_{3}}{K_{3}} x_{3}^{(6)}
\end{array}\right]
$$

The eigenvalues of $J_{6}$ are

$$
\begin{aligned}
& \lambda_{1}=-\frac{1}{2 K_{1} K_{3}}(G-\sqrt{L}), \\
& \lambda_{2}=r_{2}-c_{21} x_{1}^{(6)}-c_{23} x_{3}^{(6)}, \\
& \lambda_{3}=-\frac{1}{2 K_{1} K_{3}}(G+\sqrt{L}),
\end{aligned}
$$



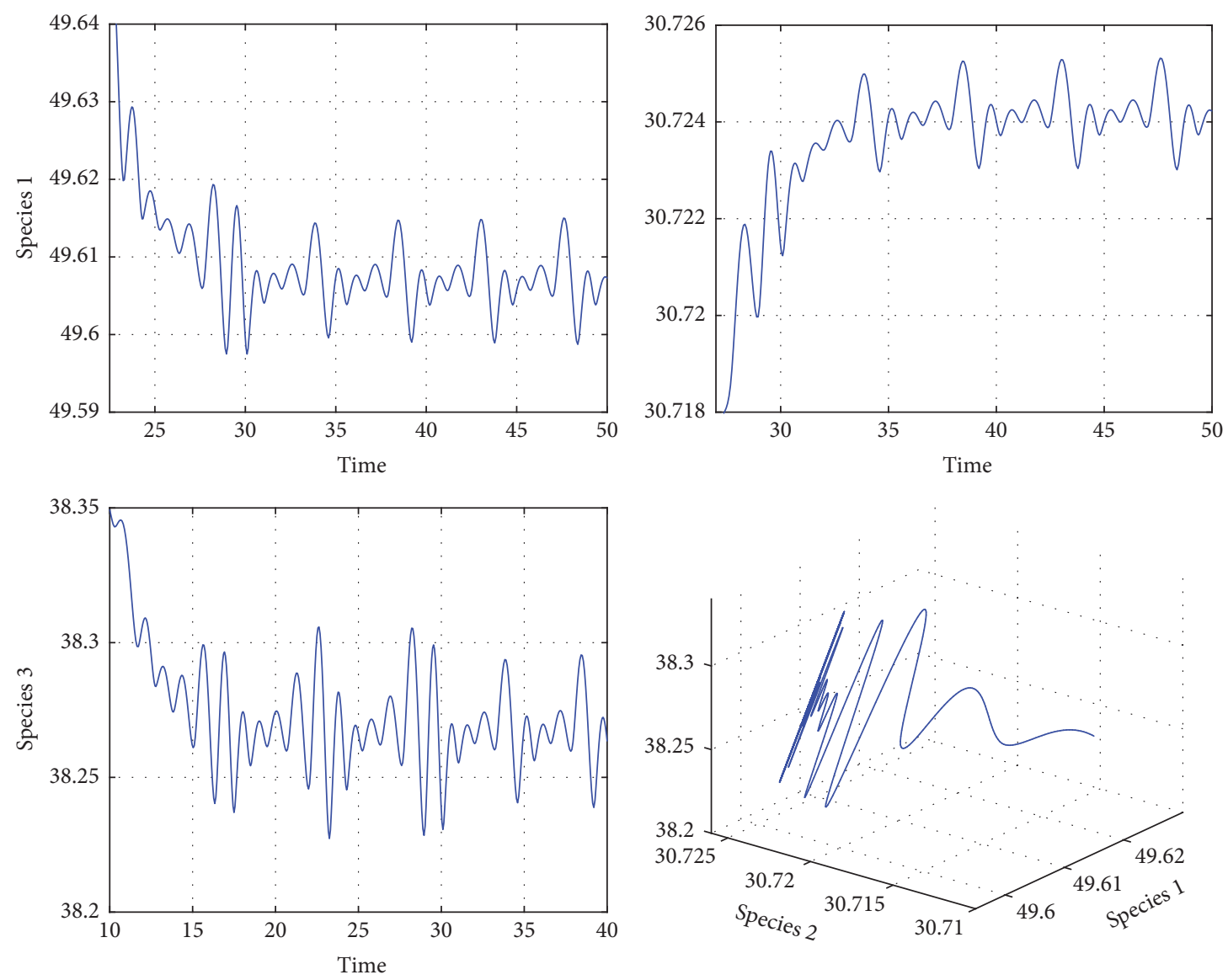

FIgURE 4: Dynamical behaviors and phase space trajectories of the three marine species.

where

$$
\begin{aligned}
& G=r_{1} x_{1}^{(6)} K_{3}+K_{1} r_{3} x_{3}^{(6)}, \\
& L=\left[r_{1} x_{1}^{(6)} K_{3}-K_{1} r_{3} x_{3}^{(6)}\right]^{2}+4 K_{1}^{2} K_{3}^{2} c_{31} x_{3}^{(6)} c_{13} x_{1}^{(6)} .
\end{aligned}
$$

If

$$
\begin{aligned}
& r_{1}>c_{13} K_{3}, \\
& r_{3}>c_{31} K_{1}
\end{aligned}
$$

then, $\lambda_{2}>0$; if not, then $\lambda_{1}>0$; therefore, point $P_{6}\left(x_{1}^{(6)}\right.$, $\left.0, x_{3}^{(6)}\right)$ is unstable.

Let $r_{1}=2, r_{2}=1, r_{3}=3, c_{12}=0.009, c_{21}=0.007, c_{13}=$ $0.008, c_{23}=0.001, c_{31}=0.002, c_{32}=0.001, K_{1}=70, K_{2}=$ $50, K_{3}=40$ in appropriate units. Figure 6 indicates the dynamical behaviors and phase space trajectory of the three marine species against time, beginning with the initial values $x(0)=59, y(0)=0.01, z(0)=38$. Following Figure 6 we can deduce that the steady state point $P_{6}$ is unstable and also tends to the point $P_{8}$.

Proposition 8. The point $P_{7}\left(0, x_{2}^{(7)}, x_{3}^{(7)}\right)$ is unstable.
Proof. The variational matrix of system (5) at the steady state $P_{7}\left(0, x_{2}^{(7)}, x_{3}^{(7)}\right)$ is

$$
J_{7}=\left[\begin{array}{ccc}
r_{1}-c_{12} x_{2}^{(7)}-c_{13} x_{3}^{(7)} & 0 & 0 \\
-c_{21} x_{2}^{(7)} & -\frac{r_{2}}{K_{2}} x_{2}^{(7)} & -c_{23} x_{2}^{(7)} \\
-c_{31} x_{3}^{(7)} & -c_{32} x_{3}^{(7)} & -\frac{r_{3}}{K_{3}} x_{3}^{(7)}
\end{array}\right] .
$$

The eigenvalues of $J_{7}$ are

$$
\begin{aligned}
& \lambda_{1}=r_{1}-c_{12} x_{2}^{(7)}-c_{13} x_{3}^{(7)}, \\
& \lambda_{2}=-\frac{1}{2 K_{2} K_{3}}(R-\sqrt{S}), \\
& \lambda_{3}=-\frac{1}{2 K_{2} K_{3}}(R+\sqrt{S}),
\end{aligned}
$$

where

$$
\begin{aligned}
& R=r_{2} x_{2}^{(7)} K_{3}+K_{2} r_{3} x_{3}^{(7)}, \\
& S=\left[r_{2} x_{2}^{(7)} K_{3}+K_{2} r_{3} x_{3}^{(7)}\right]^{2}+4 K_{2}^{2} K_{3}^{2} c_{23} x_{2}^{(7)} c_{32} x_{3}^{(7)} .
\end{aligned}
$$

If

$$
\begin{aligned}
& r_{2}>c_{23} K_{3}, \\
& r_{3}>c_{32} K_{2}
\end{aligned}
$$



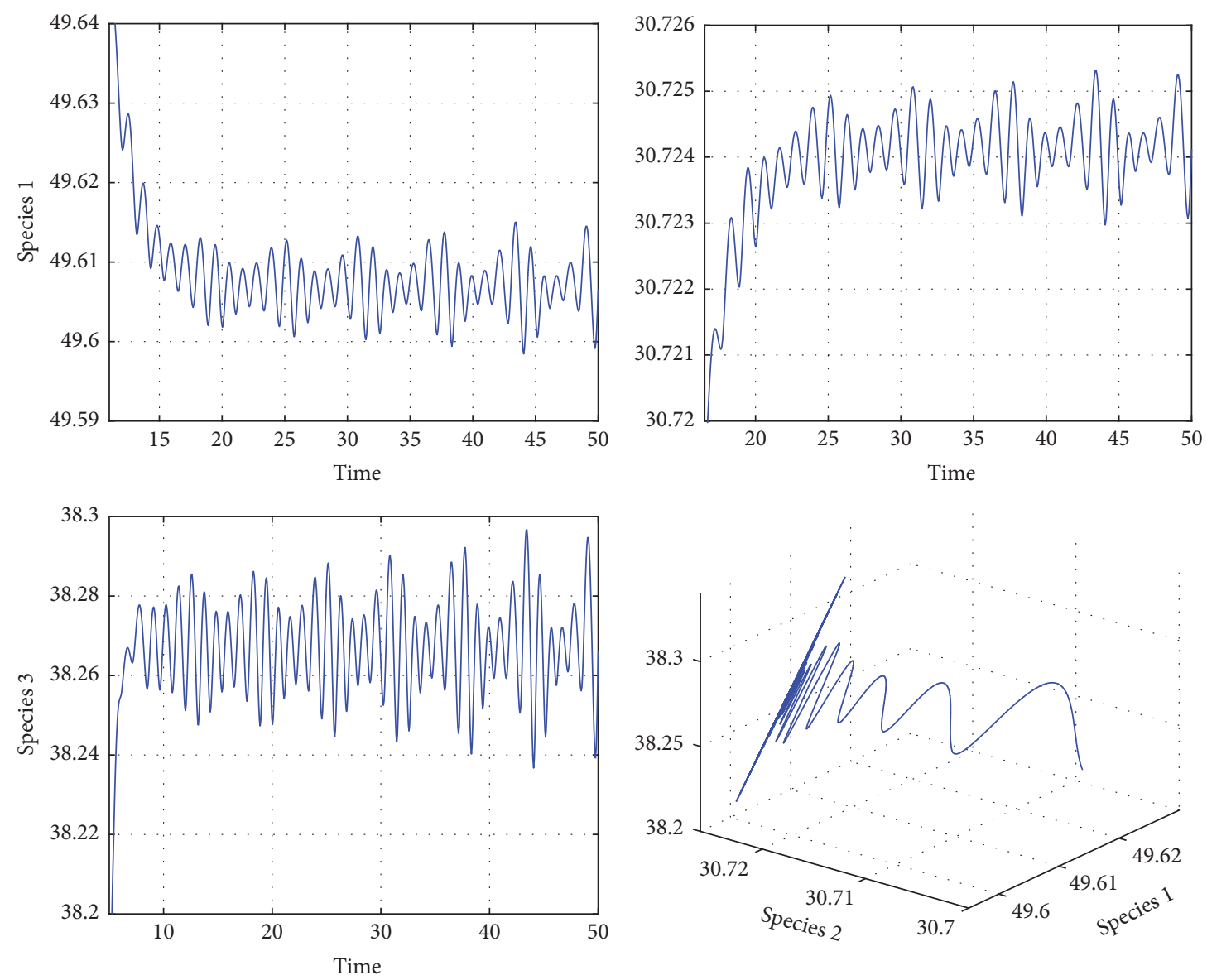

Figure 5: Dynamical behaviors and phase space trajectories of the three marine species.

then, $\lambda_{1}>0$; if not, then $\lambda_{2}>0$. Therefore, point $P_{7}\left(0, x_{2}^{(7)}\right.$, $\left.x_{3}^{(7)}\right)$ is unstable.

Let $r_{1}=2, r_{2}=1, r_{3}=3, c_{12}=0.009, c_{11}=0.007, c_{13}=$ $0.008, c_{23}=0.001, c_{31}=0.002, c_{32}=0.001, K_{1}=70, K_{2}=$ $50, K_{3}=40$ in appropriate units. Figure 7 shows the dynamical behaviors and phase space trajectory of the three marine species against time, beginning with the initial values $x(0)=$ $0.01, y(0)=48, z(0)=39$. By Figure 7 we can conclude that the steady state point $P_{7}$ is unstable and also tends to point $P_{8}$.

Theorem 9. The point $P_{8}\left(x_{1}^{*}, x_{2}^{*}, x_{3}^{*}\right)$ is locally asymptotically stable.

Proof. We proof this theorem by using Routh-Hurwitz stability criterion.

The variational matrix of system (5) in the steady state $P_{8}\left(x_{1}^{*}, x_{2}^{*}, x_{3}^{*}\right)$ is

$$
J_{8}=\left[\begin{array}{ccc}
J_{11} & -c_{12} x_{1}^{*} & -c_{13} x_{1}^{*} \\
-c_{21} x_{2}^{*} & J_{22} & -c_{23} x_{2}^{*} \\
-c_{31} x_{3}^{*} & -c_{32} x_{3}^{*} & J_{33}
\end{array}\right],
$$

where

$$
J_{11}=r_{1}\left(1-\frac{2}{K_{1}} x_{1}^{*}\right)-c_{12} x_{2}^{*}-c_{13} x_{3}^{*},
$$

$$
\begin{aligned}
& J_{22}=r_{2}\left(1-\frac{2}{K_{2}} x_{2}^{*}\right)-c_{21} x_{1}^{*}-c_{23} x_{3}^{*}, \\
& J_{33}=r_{3}\left(1-\frac{2}{K_{3}} x_{3}^{*}\right)-c_{31} x_{1}^{*}-c_{32} x_{2}^{*} .
\end{aligned}
$$

Using the fact that by (16) we have

$$
\begin{aligned}
& r_{1}\left(1-\frac{2}{K_{1}} x_{1}^{*}\right)-c_{12} x_{2}^{*}-c_{13} x_{3}^{*}=-\frac{r_{1}}{K_{1}} x_{1}^{*}, \\
& r_{2}\left(1-\frac{2}{K_{2}} x_{2}^{*}\right)-c_{21} x_{1}^{*}-c_{23} x_{3}^{*}=-\frac{r_{2}}{K_{2}} x_{2}^{*}, \\
& r_{3}\left(1-\frac{2}{K_{3}} x_{3}^{*}\right)-c_{31} x_{1}^{*}-c_{32} x_{2}^{*}=-\frac{r_{3}}{K_{3}} x_{3}^{*}
\end{aligned}
$$

then

$$
J_{8}=\left[\begin{array}{ccc}
-\frac{r_{1}}{K_{1}} x_{1}^{*} & -c_{12} x_{1}^{*} & -c_{13} x_{1}^{*} \\
-c_{21} x_{2}^{*} & -\frac{r_{2}}{K_{2}} x_{2}^{*} & -c_{23} x_{2}^{*} \\
-c_{31} x_{3}^{*} & -c_{32} x_{3}^{*} & -\frac{r_{3}}{K_{3}} x_{3}^{*}
\end{array}\right] .
$$

The characteristic polynomial of the variational matrix is

$$
P(\lambda)=a_{0} \lambda^{3}+a_{1} \lambda^{2}+a_{2} \lambda+a_{3}
$$



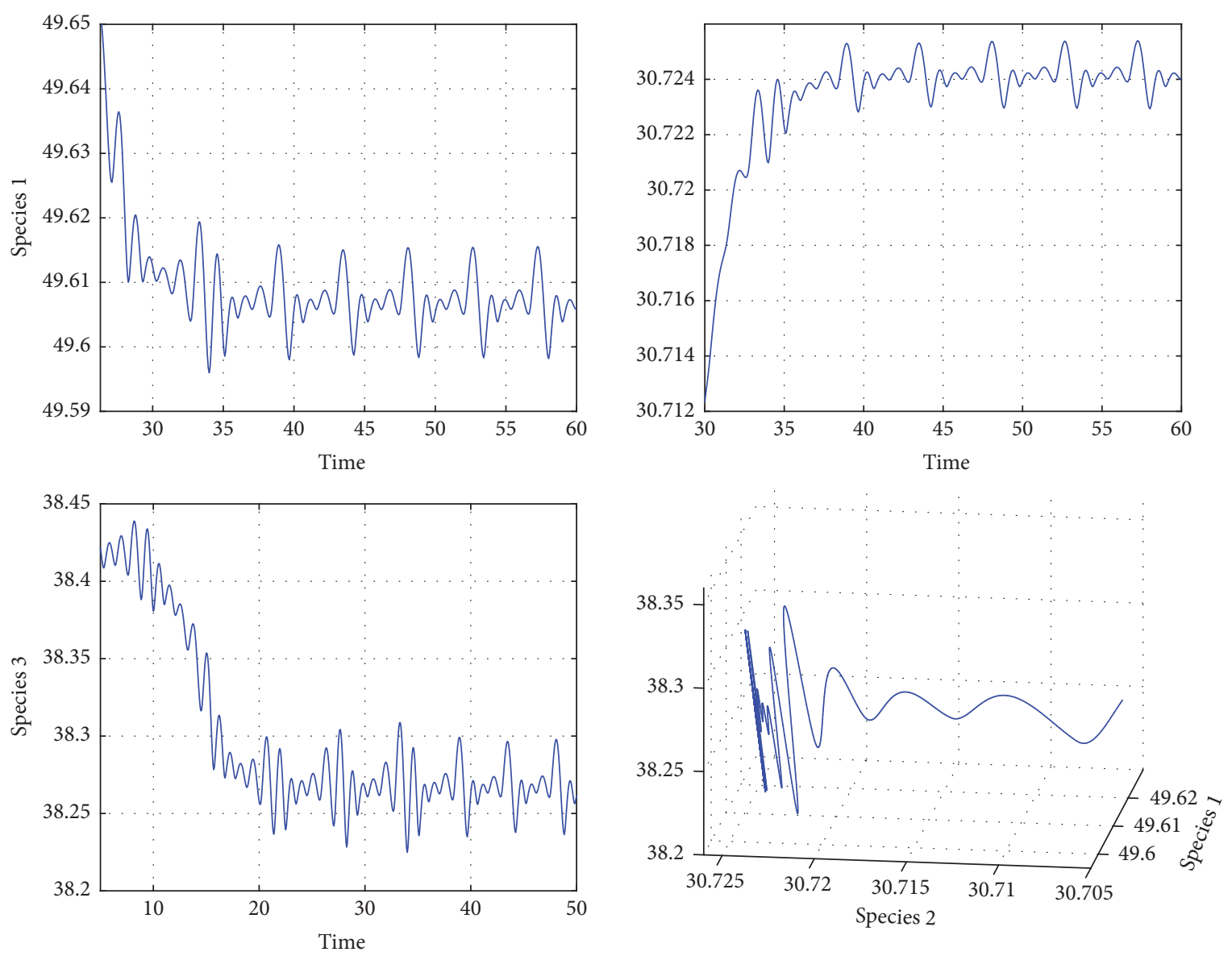

FIGURE 6: Dynamical behaviors and phase space trajectories of the three marine species.

where

$$
\begin{aligned}
a_{0}= & 1, \\
a_{1}= & \frac{r_{1}}{K_{1}} x_{1}^{*}+\frac{r_{2}}{K_{2}} x_{2}^{*}+\frac{r_{3}}{K_{3}} x_{3}^{*}, \\
a_{2}= & \frac{r_{1}}{K_{1}} x_{1}^{*} \frac{r_{2}}{K_{2}} x_{2}^{*}+\frac{r_{1}}{K_{1}} x_{1}^{*} \frac{r_{3}}{K_{3}} x_{3}^{*}+\frac{r_{2}}{K_{2}} x_{2}^{*} \frac{r_{3}}{K_{3}} x_{3}^{*} \\
& -c_{23} x_{2}^{*} c_{32} x_{3}^{*}-c_{12} x_{1}^{*} c_{21} x_{2}^{*}-c_{13} x_{1}^{*} c_{31} x_{3}^{*}, \\
a_{3}= & \frac{r_{1}}{K_{1}} x_{1}^{*} \frac{r_{2}}{K_{2}} x_{2}^{*} \frac{r_{3}}{K_{3}} x_{3}^{*}+c_{12} x_{1}^{*} c_{23} x_{2}^{*} c_{31} x_{3}^{*} \\
& +c_{13} x_{3}^{*} c_{32} x_{2}^{*} c_{21} x_{1}^{*}-c_{12} x_{1}^{*} c_{21} x_{2}^{*} \frac{r_{3}}{K_{3}} x_{3}^{*} \\
& -c_{23} x_{2}^{*} c_{32} x_{3}^{*} \frac{r_{1}}{K_{1}} x_{1}^{*}-c_{13} x_{3}^{*} c_{31} x_{1}^{*} \frac{r_{2}}{K_{2}} x_{2}^{*} ;
\end{aligned}
$$

we have $a_{i}>0, \forall i=0,1,2,3$. In fact,

(i) $a_{0}=1>0$,

(ii) $a_{1}=\left(r_{1} / K_{1}\right) x_{1}^{*}+\left(r_{2} / K_{2}\right) x_{2}^{*}+\left(r_{3} / K_{3}\right) x_{3}^{*}>0$,

(iii) using the fact that by (4) we have

$$
\begin{aligned}
& r_{1} r_{2}>c_{12} K_{2} c_{21} K_{1}, \\
& r_{2} r_{3}>c_{23} K_{3} c_{32} K_{2}, \\
& r_{1} r_{3}>c_{13} K_{3} c_{31} K_{1}
\end{aligned}
$$

(iv)

$$
\begin{aligned}
& a_{1} a_{2}-a_{0} a_{3}=\left(\frac{r_{1}}{K_{1}} x_{1}^{*}+\frac{r_{2}}{K_{2}} x_{2}^{*}+\frac{r_{3}}{K_{3}} x_{3}^{*}\right) \\
& \cdot\left(\frac{r_{1}}{K_{1}} x_{1}^{*} \frac{r_{2}}{K_{2}} x_{2}^{*}+\frac{r_{1}}{K_{1}} x_{1}^{*} \frac{r_{3}}{K_{3}} x_{3}^{*}+\frac{r_{2}}{K_{2}} x_{2}^{*} \frac{r_{3}}{K_{3}} x_{3}^{*}\right.
\end{aligned}
$$



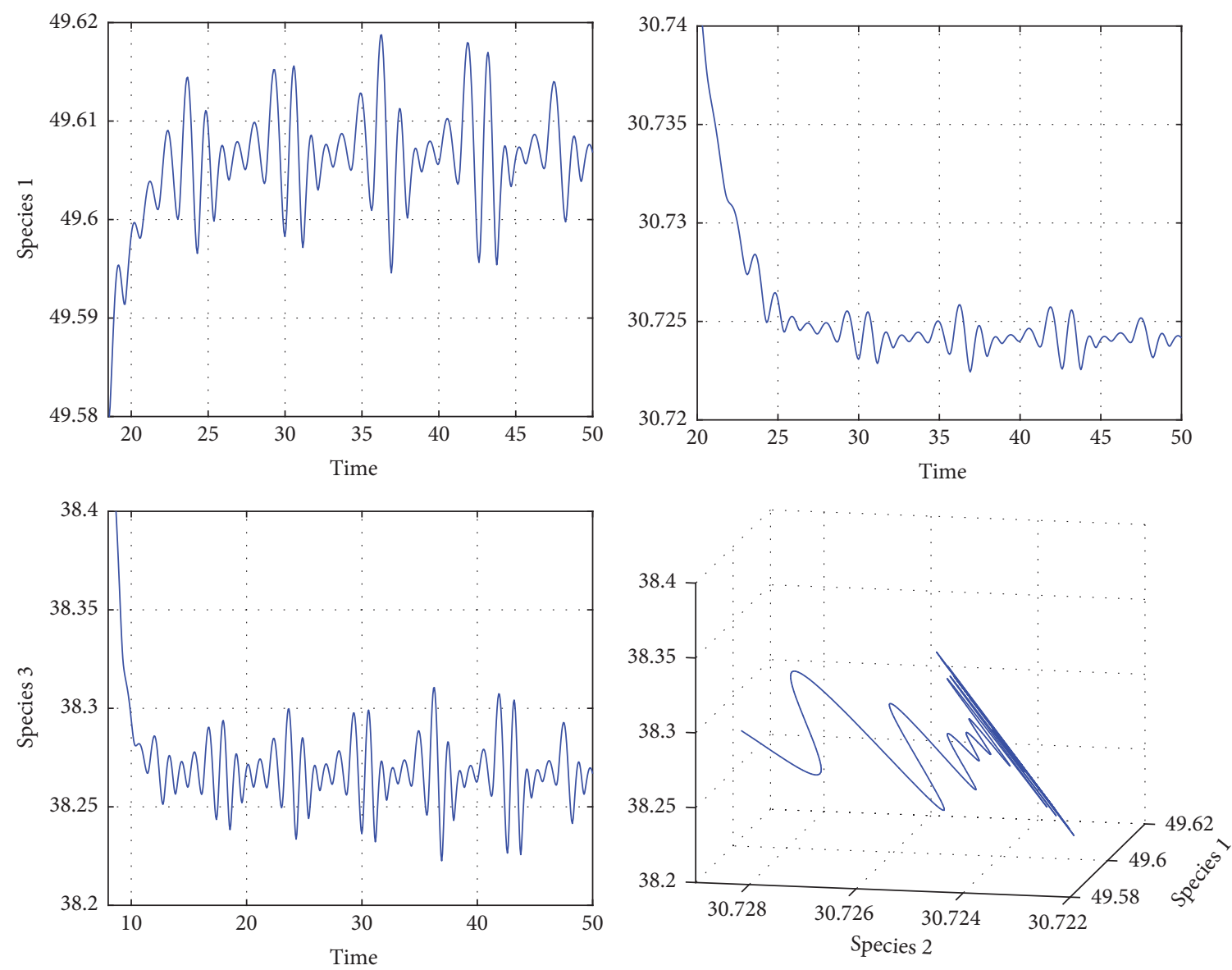

Figure 7: Dynamical behaviors and phase space trajectories of the three marine species.

$$
\begin{aligned}
& \left.-c_{23} x_{2}^{*} c_{32} x_{3}^{*}-c_{12} x_{1}^{*} c_{21} x_{2}^{*}-c_{13} x_{1}^{*} c_{31} x_{3}^{*}\right) \\
& -\left(\frac{r_{1}}{K_{1}} x_{1}^{*} \frac{r_{2}}{K_{2}} x_{2}^{*} \frac{r_{3}}{K_{3}} x_{3}^{*}+c_{12} x_{1}^{*} c_{23} x_{2}^{*} c_{31} x_{3}^{*}\right. \\
& +c_{13} x_{3}^{*} c_{32} x_{2}^{*} c_{21} x_{1}^{*}-c_{12} x_{1}^{*} c_{21} x_{2}^{*} r_{3} \frac{x_{3}^{*}}{K_{3}} \\
& \left.-c_{23} x_{2}^{*} c_{32} x_{3}^{*} \frac{r_{1}}{K_{1}} x_{1}^{*}-c_{13} x_{3}^{*} c_{31} x_{1}^{*} \frac{r_{2}}{K_{2}} x_{2}^{*}\right)=\frac{r_{1}}{K_{1}} x_{1}^{*} \\
& \cdot \frac{r_{2}}{K_{2}} x_{2}^{*} \frac{r_{3}}{K_{3}} x_{3}^{*}-c_{12} x_{1}^{*} c_{23} x_{2}^{*} c_{31} x_{3}^{*}+\frac{r_{1}}{K_{1}} x_{1}^{*} \frac{r_{2}}{K_{2}} x_{2}^{*} \\
& \cdot \frac{r_{3}}{K_{3}} x_{3}^{*}-c_{13} x_{3}^{*} c_{32} x_{2}^{*} c_{21} x_{1}^{*}+\frac{r_{1}^{2}}{K_{1}^{2}} x_{1}^{*^{2}} \frac{r_{2}}{K_{2}} x_{2}^{*}-\frac{r_{1}}{K_{1}} \\
& \cdot x_{1}^{*^{2}} c_{12} c_{21} x_{2}^{*}+\frac{r_{2}^{2}}{K_{2}^{2}} x_{2}^{*^{2}} \frac{r_{3}}{K_{3}} x_{3}^{*}-\frac{r_{2}}{K_{2}} x_{2}^{*^{2}} c_{32} c_{23} x_{3}^{*} \\
& +\frac{r_{1}^{2}}{K_{1}^{2}} x_{2}^{*^{2}} \frac{r_{3}}{K_{3}} x_{3}^{*}-\frac{r_{1}}{K_{1}} x_{1}^{*^{2}} c_{13} c_{31} x_{3}^{*}+\frac{r_{3}^{2}}{K_{3}^{2}} x_{3}^{*^{2}} \frac{r_{1}}{K_{1}} x_{1}^{*}
\end{aligned}
$$$$
-\frac{r_{3}}{K_{3}} x_{3}^{*^{2}} c_{13} c_{31} x_{1}^{*}+\frac{r_{2}^{2}}{K_{2}^{2}} x_{2}^{*^{2}} \frac{r_{1}}{K_{1}} x_{1}^{*}-\frac{r_{2}}{K_{2}} x_{2}^{*^{2}} c_{12} c_{21} x_{1}^{*}
$$$$
+\frac{r_{3}^{2}}{K_{3}^{2}} x_{3}^{*^{2}} \frac{r_{2}}{K_{2}} x_{2}^{*}-\frac{r_{3}}{K_{3}} x_{3}^{*^{2}} c_{23} c_{32} x_{2}^{*} \text {. }
$$

From (4) we deduce that

$$
a_{1} a_{2}-a_{0} a_{3}>0
$$

Then, using the Routh-Hurwitz stability criterion we conclude that the steady state point $P_{8}\left(x_{1}^{*}, x_{2}^{*}, x_{3}^{*}\right)$ is locally asymptotically stable.

$$
\text { Let } r_{1}=2, r_{2}=1, r_{3}=3, c_{12}=0.009, c_{21}=0.007, c_{13}=
$$
$0.008, c_{23}=0.001, c_{31}=0.002, c_{32}=0.001, K_{1}=70, K_{2}=$ $50, K_{3}=40$ in appropriate units. Figure 8 shows the dynamical behaviors and phase space trajectory of the three marine species against time, beginning with the initial values $x(0)=$ $49, y(0)=30, z(0)=38$. By Figure 8 one can see that the steady state point $P_{8}$ is locally asymptotically stable.

More precisely, beginning with different initial values we can confirm that the three marine species tend to point $P_{8}$, and according to the phase space trajectories given by 

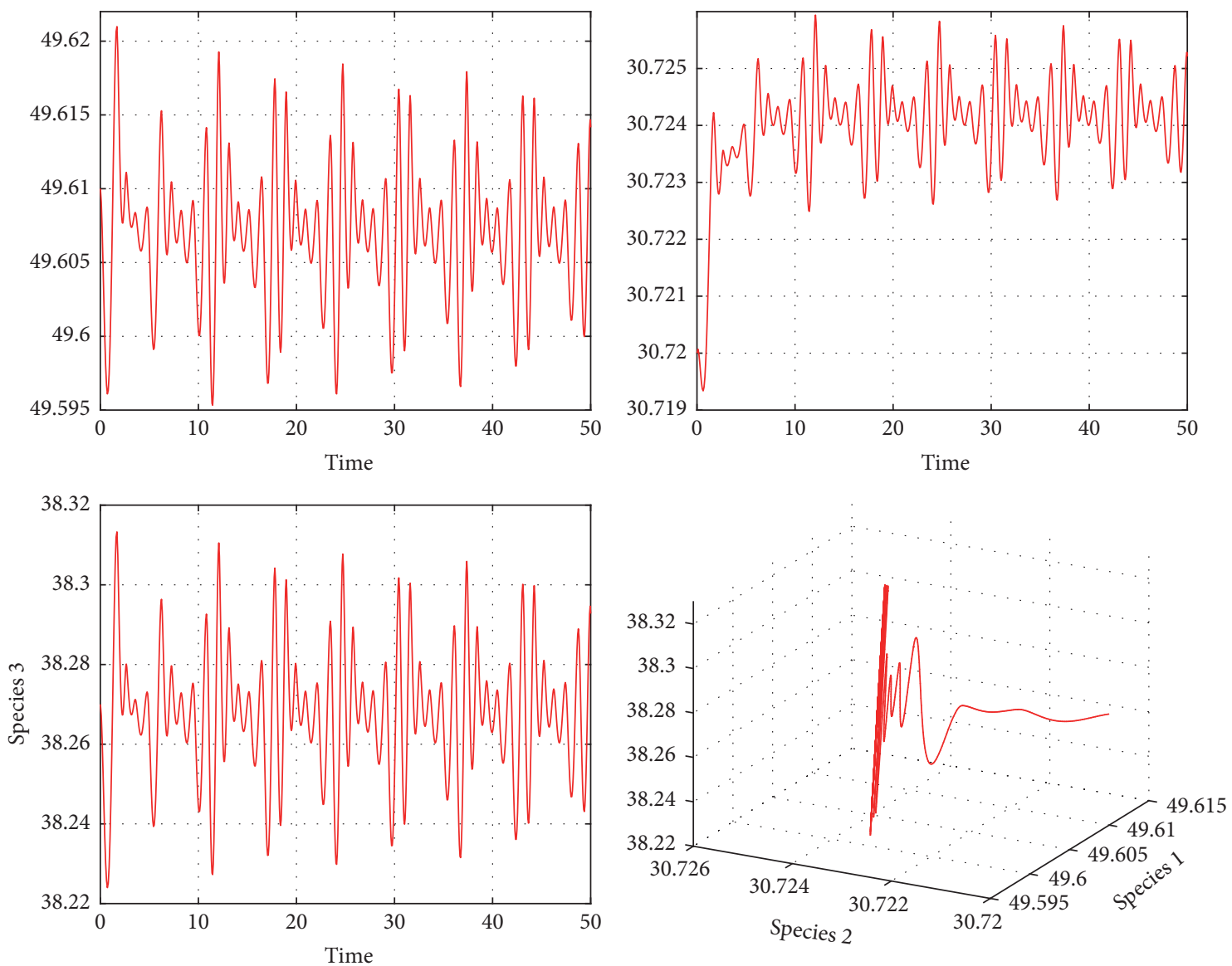

Figure 8: Dynamical behaviors and phase space trajectories of the three marine species.

Figures 1-7 we can confirm that the steady state point $P_{8}$ is a global attractor.

\section{Bioeconomic Model of Fishery}

The main purpose of this section is to define and study a bioeconomic equilibrium model for two fishermen who catch three fish populations.

More specifically, this bioeconomic model includes three parts: a biological part connecting the catch to the biomass stock, an exploitation part connecting the catch to the fishing effort, and an economic part connecting the fishing effort to the profit.

So, introducing the fishing by reducing the rate of fish population growth by the amount

$$
H_{i j}=q_{j} E_{i j} x_{j},
$$

where $H_{i j}$ is the catches of fish population $j$ by the fisherman $i, E_{i j}$ is the fishing effort to exploit a fish population $j$ by the fisherman $i$, and $q_{j}$ is the catchability coefficient of fish population $j$, the model for the evolution of fish populations is given by the following mathematical system of equations:

$$
\dot{x}_{1}=r_{1} x_{1}\left(1-\frac{x_{1}}{K_{1}}\right)-c_{12} x_{1} x_{2}-c_{13} x_{1} x_{3}-q_{1} E_{1} x_{1},
$$

$$
\begin{aligned}
& \dot{x}_{2}=r_{2} x_{2}\left(1-\frac{x_{2}}{K_{2}}\right)-c_{21} x_{1} x_{2}-c_{23} x_{2} x_{3}-q_{2} E_{2} x_{2}, \\
& \dot{x}_{3}=r_{3} x_{3}\left(1-\frac{x_{3}}{K_{3}}\right)-c_{31} x_{1} x_{3}-c_{32} x_{2} x_{3}-q_{3} E_{3} x_{3} .
\end{aligned}
$$

On one hand, we denote by $H_{j}=H_{1 j}+H_{2 j}$ the total catches of species $j$ by all fishermen; on the other hand, we denote by $E_{j}=E_{1 j}+E_{2 j}$ the total fishing effort dedicated to species $j$ by all fishermen, and we denote by $E^{(i)}=\left(E_{i 1}, E_{i 2}, E_{i 3}\right)^{T}$ the vector fishing effort which must be provided by the fisherman $i$ to catch the three species.

In what follows of this paper, the product of two vectors $\alpha \in \mathbb{R}^{3}$ and $\beta \in \mathbb{R}^{3}$ is the vector noted by $\alpha \beta$ or $\beta \alpha$ and is defined by

$$
\alpha \beta:=\left(\alpha_{1} y_{1}, \alpha_{2} y_{2}, \alpha_{3} y_{3}\right)^{T} \in \mathbb{R}^{3} \text {. }
$$

The scalar product is noted by $\alpha^{T} \beta$. The division of the vector $\alpha \in \mathbb{R}^{3}$ and the not null vector $\beta \in \mathbb{R}^{3}$ (i.e., $\beta_{i} \neq 0, \forall i=1,2,3$ ) is the vector noted by $\alpha / \beta$ and is defined by

$$
\frac{\alpha}{\beta}:=\left(\frac{\alpha_{1}}{\beta_{1}}, \frac{\alpha_{2}}{\beta_{2}}, \frac{\alpha_{3}}{\beta_{3}}\right)^{T} \in \mathbb{R}^{3} .
$$


The product of the vector $\alpha \in \mathbb{R}^{3}$ and the matrix $A \in \mathbb{R}^{3 \times 3}$ is noted by $\alpha A$ and is defined by

$$
\alpha A:=\operatorname{diag}(\alpha) \cdot A \in \mathbb{R}^{3 \times 3} .
$$

Now we give the expression of biomass as a function of fishing effort.

The biomasses at biological equilibrium are the solutions of the system

$$
\begin{aligned}
& r_{1}\left(1-\frac{x_{1}}{K_{1}}\right)=c_{12} x_{2}+c_{13} x_{3}+q_{1} E_{1}, \\
& r_{2}\left(1-\frac{x_{2}}{K_{2}}\right)=c_{21} x_{1}+c_{23} x_{3}+q_{2} E_{2}, \\
& r_{3}\left(1-\frac{x_{3}}{K_{3}}\right)=c_{31} x_{1}+c_{32} x_{2}+q_{3} E_{3} .
\end{aligned}
$$

The solutions of this system are given by

$$
\begin{aligned}
& x_{1}=a_{11} E_{1}+a_{12} E_{2}+a_{13} E_{3}+x_{1}^{*}, \\
& x_{2}=a_{21} E_{1}+a_{22} E_{2}+a_{23} E_{3}+x_{2}^{*}, \\
& x_{3}=a_{31} E_{1}+a_{32} E_{2}+a_{33} E_{3}+x_{3}^{*},
\end{aligned}
$$

where

$$
\begin{aligned}
& a_{11}=\frac{K_{1}\left(c_{32} K_{2} K_{3} c_{23} q_{1}-r_{3} r_{2} q_{1}\right)}{\Delta}, \\
& a_{12}=\frac{K_{1}\left(-c_{32} K_{2} q_{2} c_{13} K_{3}+K_{2} q_{2} c_{12} r_{3}\right)}{\Delta}, \\
& a_{13}=\frac{K_{1}\left(-K_{2} K_{3} c_{23} c_{12} q_{3}+q_{3} r_{2} c_{13} K_{3}\right)}{\Delta}, \\
& a_{21}=\frac{K_{2}\left(-K_{3} c_{23} q_{1} K_{1} c_{31}+K_{1} c_{21} r_{3} q_{1}\right)}{\Delta}, \\
& a_{22}=\frac{K_{2}\left(q_{2} c_{13} K_{1} K_{3} c_{31}-q_{2} r_{1} r_{3}\right)}{\Delta}, \\
& a_{23}=\frac{K_{2}\left(K_{3} c_{23} r_{1} q_{3}-K_{1} c_{21} q_{3} c_{13} K_{3}\right)}{\Delta}, \\
& a_{31}=\frac{K_{3}\left(-q_{1} K_{1} c_{32} K_{2} c_{21}+q_{1} K_{1} r_{2} c_{31}\right)}{\Delta}, \\
& a_{32}=\frac{K_{3}\left(r_{1} c_{32} K_{2} q_{2}-c_{12} K_{1} K_{2} q_{2} c_{31}\right)}{\Delta}, \\
& a_{33}=\frac{K_{3}\left(c_{12} K_{1} K_{2} c_{21} q_{3}-r_{1} r_{2} q_{3}\right)}{\Delta}
\end{aligned}
$$

or in matrix form $X=-A E+X^{*}$, where $A=\left(-a_{i j}\right)_{1 \leq i, j \leq 3}$, $E=\left(E_{1}, E_{2}, E_{3}\right)^{T}$, and $X^{*}=\left(x_{1}^{*}, x_{2}^{*}, x_{3}^{*}\right)^{T}$.

3.1. Expression of the Total Revenue. It is interesting to note that there exist many different variables that affect the fish price; in this paper, we will consider that the price of the fish population depends on the quantity harvested; specifically we assumed that the price of the marine species increases with the decreasing harvest and the price of the marine species decreases with the increase of the harvest, but the minimum price is equal to a fixed positive constant. More precisely, the price of marine species $j$ exploited by the fisherman $i$ is given by $p_{i j}=a_{j} / H_{i j}+p_{0 j}$, where $a_{j}$ and $p_{0 j}$ are given positive parameters for all $j=1,2,3$. Under these more realistic assumptions the total revenue of the fisherman $i$ is

$$
\begin{aligned}
(\mathrm{TR})_{i}= & p_{i 1} H_{i 1}+p_{i 2} H_{i 2}+p_{i 3} H_{i 3} \\
= & \left(\frac{a_{1}}{H_{i 1}}+p_{01}\right) H_{i 1}+\left(\frac{a_{2}}{H_{i 2}}+p_{02}\right) H_{i 2} \\
& +\left(\frac{a_{3}}{H_{i 3}}+p_{03}\right) H_{i 3} \\
= & p_{01} H_{i 1}+p_{02} H_{i 2}+p_{03} H_{i 3}+\sum_{k=1}^{3} a_{k} \\
= & p_{01} q_{1} E_{i 1} x_{1}+p_{02} q_{2} E_{i 2} x_{2}+p_{03} q_{3} E_{i 3} x_{3} \\
& +\sum_{k=1}^{3} a_{k}=\left\langle P_{0}, q E^{(i)} X\right\rangle+\sum_{k=1}^{3} a_{k} \\
= & \left\langle P_{0}, q E^{(i)}\left(-A E+X^{*}\right)\right\rangle+\sum_{k=1}^{3} a_{k} \\
= & \left\langle P_{0}, q E^{(i)}\left(-A \sum_{i=1}^{2} E^{(i)}+X^{*}\right)\right\rangle+\sum_{k=1}^{3} a_{k} \\
= & \left\langle E^{(i)}, q P_{0}\left(-A \sum_{i=1}^{2} E^{(i)}+X^{*}\right)\right\rangle+\sum_{k=1}^{3} a_{k},
\end{aligned}
$$

so,

$$
\begin{aligned}
(\mathrm{TR})_{i}= & \left\langle E^{(i)},-P_{0} q A E^{(i)}\right\rangle \\
& +\left\langle E^{(i)}, P_{0} q x X^{*}-P_{0} q A \mathrm{E}^{(j)}\right\rangle+\sum_{k=1}^{3} a_{k},
\end{aligned}
$$

where $P_{0}=\operatorname{diag}\left(p_{0}\right)$.

3.2. Expression of the Total Effort Cost. In accordance with many standard fisheries models, we consider that expression of the total effort cost is

$$
(\mathrm{TC})_{i}=\left\langle c^{(i)}, E^{(i)}\right\rangle,
$$

where $c^{(i)}$ is the constant cost per unit of harvesting and $E^{(i)}$ is the total effort of the fisherman $i$.

3.3. Expression of the Profit. The profit of each fisherman $\pi_{i}$ is equal to total revenue $(\mathrm{TR})_{i}$ minus total cost $(\mathrm{TC})_{i}$; it is represented by the following function:

$$
\begin{aligned}
\pi_{i}\left(E^{(i)}\right) & =(\mathrm{TR})_{i}-(\mathrm{TC})_{i} \\
& =\left\langle E^{(i)},-P_{0} q A E^{(i)}\right\rangle
\end{aligned}
$$




$$
\begin{aligned}
& +\left\langle E^{(i)}, P_{0} q X^{*}-P_{0} q A E^{(j)}\right\rangle+\sum_{k=1}^{3} a_{k} \\
& -\left\langle c^{(i)}, E^{(i)}\right\rangle \\
& =\left\langle E^{(i)},-P_{0} q A E^{(i)}\right\rangle \\
& +\left\langle E^{(i)}, P_{0} q X^{*}-c^{(i)}-P_{0} q A E^{(j)}\right\rangle+\sum_{k=1}^{3} a_{k} .
\end{aligned}
$$

3.4. Constraints of the Model. The biological model has a meaning if and only if the biomass of all the marine species are strictly positive, then we have

$$
X=-A E+X^{*} \geq X_{0}>0 .
$$

In other words, for the fisherman $i$,

$$
A E^{(i)} \leq-A E^{(j)}+X^{*} .
$$

3.5. Nash Equilibrium Problem. The problem of determining the fishing effort that maximizes the profit of each fisherman leads to a Nash equilibrium problem. By definition a Nash equilibrium exists when there is no unilateral profitable deviation from any of the fishermen involved. In other words, no fisherman would take a different action as long as every other fisherman remains the same. This problem can be translated into the following two mathematical problems.

The first fisherman must solve the problem $\left(P_{1}\right)$ :

$$
\begin{aligned}
\max & \pi_{1}\left(E^{(1)}\right) \\
= & \left\langle E^{(1)},-P_{0} q A E^{(1)}+P_{0} q X^{*}-c^{(1)}-P_{0} q A E^{(2)}\right\rangle \\
& +\sum_{k=1}^{3} a_{k}
\end{aligned}
$$

subject to $A E^{(1)} \leq-A E^{(2)}+X^{*}$

$$
\begin{aligned}
& E^{(1)} \geq 0 \\
& E^{(2)} \text { given }
\end{aligned}
$$

and the second fisherman must solve the problem $\left(P_{2}\right)$ :

$$
\begin{aligned}
\max & \pi_{2}\left(E^{(2)}\right) \\
= & \left\langle E^{(2)},-P_{0} q A E^{(2)}+P_{0} q X^{*}-c^{(2)}-P_{0} q A E^{(1)}\right\rangle \\
& +\sum_{k=1}^{3} a_{k}
\end{aligned}
$$

subject to $A E^{(2)} \leq-A E^{(1)}+X^{*}$

$$
\begin{aligned}
& E^{(2)} \geq 0 \\
& E^{(1)} \text { given. }
\end{aligned}
$$

The point $\left(E^{(1)}, E^{(2)}\right)$ is called Nash equilibrium point if and only if $E^{(1)}$ is a solution of problem $\left(P_{1}\right)$ for $E^{(2)}$ given, and $E^{(2)}$ is solution of problem $\left(P_{2}\right)$ for $E^{(1)}$ given.
The essential conditions of Karush-Kuhn-Tucker applied to the problem $\left(P_{1}\right)$ confirm that if $E^{(1)}$ is a solution of the problem $\left(P_{1}\right)$ then there exist constants $u^{(1)} \in \mathbb{R}_{+}^{3}, v^{(1)} \in \mathbb{R}_{+}^{3}$, and $\lambda^{(1)} \in \mathbb{R}_{+}^{3}$ such that

$$
\begin{aligned}
& 2 P_{0} q A E^{(1)}+c^{(1)}-P_{0} q X^{*}+P_{0} q A E^{(2)}-u^{(1)} \\
& \quad+A^{T} \lambda^{(1)}=0 \\
& A E^{(1)}+v^{(1)}=-A E^{(2)}+X^{*}, \\
& \left\langle u^{(1)}, E^{(1)}\right\rangle=\left\langle\lambda^{(1)}, v^{(1)}\right\rangle=0 .
\end{aligned}
$$

In the same way, the essential conditions of KarushKuhn-Tucker applied to the problem $\left(P_{2}\right)$ confirm that if $E^{(2)}$ is a solution of the problem $\left(P_{2}\right)$ then there exist constants $u^{(2)} \in \mathbb{R}_{+}^{3}, v^{(2)} \in \mathbb{R}_{+}^{3}$, and $\lambda^{(2)} \in \mathbb{R}_{+}^{3}$ such that

$$
\begin{aligned}
& 2 P_{0} q A E^{(2)}+c^{(2)}-P_{0} q X^{*}+P_{0} q A E^{(1)}-u^{(2)} \\
& \quad+A^{T} \lambda^{(2)}=0 \\
& A E^{(2)}+v^{(2)}=-A E^{(1)}+X^{*} \\
& \left\langle u^{(2)}, E^{(2)}\right\rangle=\left\langle\lambda^{(2)}, v^{(2)}\right\rangle=0
\end{aligned}
$$

we remark that (KKT1) and (KKT2) lead to the following system:

$$
\begin{aligned}
& u^{(1)}= 2 P_{0} q A E^{(1)}+c^{(1)}-P_{0} q X^{*} \\
&+P_{0} q A E^{(2)}+A^{T} \lambda^{(1)}, \\
& u^{(2)}= 2 P_{0} q A E^{(2)}+c^{(2)}-P_{0} q X^{*} \\
&+P_{0} q A E^{(1)}+A^{T} \lambda^{(2)}, \\
& v^{(1)}=-A E^{(1)}-A E^{(2)}+X^{*}, \\
& v^{(2)}=-A E^{(1)}-A E^{(2)}+X^{*}, \\
&\left\langle u^{(i)}, E^{(i)}\right\rangle=\left\langle\lambda^{(i)}, v^{(i)}\right\rangle=0 \quad \forall i=1,2, \\
& E^{(i)}, u^{(i)}, \lambda^{(i)}, v^{(i)} \geq 0 \quad \forall i=1,2 .
\end{aligned}
$$

To maintain the biodiversity of species, it is natural to assume that all biomasses remain strictly positive; that is, $x_{j}>0$ for all $j=1,2,3$; therefore $v^{(1)}=v^{(2)}>0$.

As the scalar product of $\left(\lambda^{(i)}\right)_{i=1,2}$ and $\left(v^{(i)}\right)_{i=1,2}$ is zero, $\lambda^{(i)}=0$ for all $i=1,2$. So, we denote $v=v^{(1)}=v^{(2)}$. Then we have the following expressions:

$$
\begin{aligned}
u^{(1)} & =2 P_{0} q A E^{(1)}+P_{0} q A E^{(2)}+c^{(1)}-P_{0} q X^{*}, \\
u^{(2)} & =2 P_{0} q A E^{(2)}+P_{0} q A E^{(1)}+c^{(2)}-P_{0} q X^{*}, \\
v & =-A E^{(1)}-A E^{(2)}+X^{*},
\end{aligned}
$$

$$
\begin{gathered}
\left\langle u^{(i)}, E^{(i)}\right\rangle=0 \quad \forall i=1,2, \\
E^{(i)}, u^{(i)}, v \geq 0 \quad \forall i=1,2 .
\end{gathered}
$$


Thus

$$
\begin{aligned}
\left(\begin{array}{c}
u^{(1)} \\
u^{(2)} \\
v
\end{array}\right)= & {\left[\begin{array}{ccc}
2 P_{0} q A & P_{0} q A & A^{T} \\
P_{0} q A & 2 P_{0} q A & 0 \\
-A & -A & 0
\end{array}\right]\left(\begin{array}{c}
E^{(1)} \\
E^{(2)} \\
0
\end{array}\right) } \\
& +\left(\begin{array}{c}
c^{(1)}-P_{0} q X^{*} \\
c^{(2)}-P_{0} q X^{*} \\
X^{*}
\end{array}\right) .
\end{aligned}
$$

3.6. Linear Complementarity Problem. We denote

$$
\begin{aligned}
z & =\left(\begin{array}{c}
E^{(1)} \\
E^{(2)} \\
0
\end{array}\right), \\
w & =\left(\begin{array}{c}
u^{(1)} \\
u^{(2)} \\
v
\end{array}\right), \\
M & =\left(\begin{array}{ccc}
2 P_{0} q A & P_{0} q A & A^{T} \\
P_{0} q A & 2 P_{0} q A & 0 \\
-A & -A & 0
\end{array}\right], \\
b & =\left(\begin{array}{c}
c^{(1)}-P_{0} q X^{*} \\
c^{(2)}-P_{0} q X^{*} \\
X^{*}
\end{array}\right) .
\end{aligned}
$$

The Nash equilibrium problem is equivalent to the Linear Complementarity Problem $\operatorname{LCP}(M, b)$. Find vectors $z, w \in$ $\mathbb{R}^{6}$ such that

$$
\begin{aligned}
w & =M z+b \geq 0, \\
z, w & \geq 0 \\
z^{T} w & =0 .
\end{aligned}
$$

The following proposition confirms that $\operatorname{LCP}(M, b)$ has a unique solution.

Proposition 10. The matrix

$$
M=\left[\begin{array}{ccc}
2 P_{0} q A & P_{0} q A & A^{T} \\
P_{0} q A & 2 P_{0} q A & 0 \\
-A & -A & 0
\end{array}\right]
$$

is $P$-matrix.

Proof. We have $a_{i i}<0$ for all $i=1,2,3$ and $\Delta>0$ so, if we note by $\left(M_{i}\right)_{i=1, \ldots, 9}$ the submatrix of $M$, we obtain

$$
\begin{aligned}
& \operatorname{det}\left(M_{1}\right)=-2 p_{01} q_{1} a_{11}>0, \\
& \operatorname{det}\left(M_{2}\right)=4 p_{01} q_{1} p_{02} q_{2} q_{2} q_{1} K_{1} r_{3} q_{2} K_{2} \Delta>0, \\
& \operatorname{det}\left(M_{3}\right)=8 p_{01} q_{1} p_{02} q_{2} p_{3} q_{3} q_{3} K_{3} q_{1} K_{1} q_{2} K_{2} \Delta^{2}>0,
\end{aligned}
$$

$$
\begin{aligned}
& \operatorname{det}\left(M_{4}\right)=-12 a_{11} p_{01}^{2} q_{1}^{2} p_{02} q_{2} p_{03} q_{3} q_{3} K_{3} q_{1} K_{1} q_{2} K_{2} \Delta^{2} \\
& >0 \\
& \operatorname{det}\left(M_{5}\right) \\
& =18 p_{01}^{2} q_{1}^{2} p_{02}^{2} q_{2}^{2} p_{03} q_{3} q_{1} K_{1} r_{3} q_{2} K_{2} q_{3} K_{3} q_{1} K_{1} q_{2} K_{2} \Delta^{3} \\
& >0, \\
& \operatorname{det}\left(M_{6}\right)=27 p_{01}^{2} q_{1}^{2} p_{02}^{2} q_{2}^{2} p_{03}^{2} q_{3}^{2}\left(q_{3} K_{3} q_{1} K_{1} q_{2} K_{2} \Delta^{2}\right)^{2} \\
& >0, \\
& \operatorname{det}\left(M_{7}\right)=-9 p_{01} q_{1} p_{02}^{2} q_{2}^{2} p_{03}^{2} q_{3}^{2} a_{11}\left(q_{3} K_{3} q_{1} K_{1} q_{2} K_{2} \Delta^{2}\right)^{2} \\
& >0, \\
& \operatorname{det}\left(M_{8}\right) \\
& =3 p_{01} q_{1} p_{02} q_{2} p_{03}^{2} q_{3}^{2} q_{1} K_{1} r_{3} q_{2} K_{2} \Delta\left(q_{3} K_{3} q_{1} K_{1} q_{2} K_{2} \Delta^{2}\right)^{2} \\
& >0, \\
& \operatorname{det}(M)=p_{01} q_{1} p_{02} q_{2} p_{03} q_{3}\left(q_{3} K_{3} q_{1} K_{1} q_{2} K_{2} \Delta^{2}\right)^{2}>0 .
\end{aligned}
$$

Then, the matrix $M$ is $P$-matrix and therefore the linear complementarity problem $\operatorname{LCP}(M, b)$ admits one and only one solution.

The unique solution of $\operatorname{LCP}(M, b)$ represents the Nash equilibrium point of our problem and it is given by

$$
\begin{aligned}
& E^{(1)}=\frac{1}{3} A^{-1}\left(X^{*}-\frac{c^{(1)}}{P_{0} q}\right), \\
& E^{(2)}=\frac{1}{3} A^{-1}\left(X^{*}-\frac{c^{(2)}}{P_{0} q}\right),
\end{aligned}
$$

where

$$
A^{-1}=\left[\begin{array}{ccc}
\frac{r_{1}}{K_{1} q_{1}} & \frac{c_{12}}{q_{1}} & \frac{c_{13}}{q_{1}} \\
\frac{c_{21}}{q_{2}} & \frac{r_{2}}{K_{2} q_{2}} & \frac{c_{23}}{q_{2}} \\
\frac{c_{31}}{q_{3}} & \frac{c_{32}}{q_{3}} & \frac{r_{3}}{K_{3} q_{3}}
\end{array}\right] .
$$

Then, the fishing effort that maximizes the profit of the first fisherman for catching the first species is

$$
\begin{aligned}
E_{11} & =\frac{1}{3}\left[\frac{r_{1}}{K_{1} q_{1}}\left(x_{1}^{*}-\frac{c_{1}}{P_{01} q_{1}}\right)+\frac{c_{12}}{q_{1}}\left(x_{2}^{*}-\frac{c_{1}}{P_{02} q_{2}}\right)\right. \\
& \left.+\frac{c_{13}}{q_{1}}\left(x_{3}^{*}-\frac{c_{1}}{P_{03} q_{3}}\right)\right] ;
\end{aligned}
$$


the fishing effort that maximizes the profit of the first fisherman for catching the second species is

$$
\begin{aligned}
E_{12} & =\frac{1}{3}\left[\frac{r_{2}}{K_{2} q_{2}}\left(x_{2}^{*}-\frac{c_{1}}{P_{02} q_{2}}\right)+\frac{c_{21}}{q_{2}}\left(x_{1}^{*}-\frac{c_{1}}{P_{01} q_{1}}\right)\right. \\
& \left.+\frac{c_{23}}{q_{2}}\left(x_{3}^{*}-\frac{c_{1}}{P_{03} q_{3}}\right)\right] ;
\end{aligned}
$$

the fishing effort that maximizes the profit of the first fisherman for catching the third species is

$$
\begin{aligned}
E_{13} & =\frac{1}{3}\left[\frac{r_{3}}{K_{3} q_{3}}\left(x_{3}^{*}-\frac{c_{1}}{P_{03} q_{3}}\right)+\frac{c_{31}}{q_{3}}\left(x_{1}^{*}-\frac{c_{1}}{P_{01} q_{1}}\right)\right. \\
& \left.+\frac{c_{32}}{q_{3}}\left(x_{2}^{*}-\frac{c_{1}}{P_{02} q_{2}}\right)\right] .
\end{aligned}
$$

The fishing effort that maximizes the profit of the second fisherman for catching the first species is

$$
\begin{aligned}
E_{21} & =\frac{1}{3}\left[\frac{r_{1}}{K_{1} q_{1}}\left(x_{1}^{*}-\frac{c_{2}}{P_{01} q_{1}}\right)+\frac{c_{12}}{q_{1}}\left(x_{2}^{*}-\frac{c_{2}}{P_{02} q_{2}}\right)\right. \\
& \left.+\frac{c_{13}}{q_{1}}\left(x_{3}^{*}-\frac{c_{2}}{P_{03} q_{3}}\right)\right] ;
\end{aligned}
$$

the fishing effort that maximizes the profit of the second fisherman for catching the second species is

$$
\begin{aligned}
E_{22} & =\frac{1}{3}\left[\frac{r_{2}}{K_{2} q_{2}}\left(x_{2}^{*}-\frac{c_{2}}{P_{02} q_{2}}\right)+\frac{c_{21}}{q_{2}}\left(x_{1}^{*}-\frac{c_{2}}{P_{01} q_{1}}\right)\right. \\
& \left.+\frac{c_{23}}{q_{2}}\left(x_{3}^{*}-\frac{c_{2}}{P_{03} q_{3}}\right)\right] ;
\end{aligned}
$$

the fishing effort that maximizes the profit of the second fisherman for catching the third species is

$$
\begin{aligned}
E_{23} & =\frac{1}{3}\left[\frac{r_{3}}{K_{3} q_{3}}\left(x_{3}^{*}-\frac{c_{2}}{P_{03} q^{3}}\right)+\frac{c_{31}}{q_{3}}\left(x_{1}^{*}-\frac{c_{2}}{P_{01} q_{1}}\right)\right. \\
& \left.+\frac{c_{32}}{q_{3}}\left(x_{2}^{*}-\frac{c_{2}}{P_{02} q_{2}}\right)\right] .
\end{aligned}
$$

\section{Numerical Simulations and Discussion of the Results}

In this section, we take as case of study two fishermen who catch three fish species competing with each other for space or food. In order to assure the existence and stability of the locally asymptotically stable state of the three fish populations, we consider the parameters of the model system (5) as shown in Table 1.

Let us consider the economic parameters such as that shown in Table 2.

Using the parameters cited in Tables 1 and 2, thereafter we will see how changes in the minimum prices can affect effort fishing, catches, and profit.
TABLE 1: Characteristics of the three marine species.

\begin{tabular}{lcc}
\hline Species 1 & Species 2 & Species 3 \\
\hline$r_{1}=0,5$ & $r_{2}=0,3$ & $r_{3}=0,2$ \\
$K_{1}=1000$ & $K_{2}=700$ & $K_{3}=600$ \\
$c_{12}=2 \cdot 10^{-4}$ & $c_{21}=10^{-5}$ & $c_{31}=10^{-4}$ \\
$c_{13}=3 \cdot 10^{-4}$ & $c_{23}=2 \cdot 10^{-5}$ & $c_{32}=10^{-4}$ \\
\hline
\end{tabular}

TABLE 2: Economic parameters of the model.

\begin{tabular}{lcc}
\hline Species 1 & Species 2 & Species 3 \\
\hline$a_{1}=0,1$ & $a_{2}=0,2$ & $a_{3}=0,3$ \\
$p_{01}=1$ & $p_{02}=2$ & $p_{03}=3$ \\
$q_{1}=0,1$ & $q_{2}=0,02$ & $q_{3}=0,004$ \\
$c_{1}=0,1$ & $c_{1}=0,1$ & $c_{1}=0,1$ \\
$c_{2}=0,2$ & $c_{2}=0,2$ & $c_{2}=0,2$ \\
\hline
\end{tabular}

TABLE 3: The influence of the price on the fishing effort.

\begin{tabular}{lcccc}
\hline$p_{01}$ & $p_{02}$ & $p_{03}$ & $E_{1}$ & $E_{2}$ \\
\hline 1 & 2 & 3 & 17,0451 & 16,5151 \\
11 & 17 & 23 & 17,5943 & 17,5314 \\
16 & 27 & 48 & 17,6383 & 17,6073 \\
31 & 47 & 78 & 17,6552 & 17,6363 \\
51 & 70 & 108 & 17,6627 & 17,6492 \\
84 & 101 & 273 & 17,6734 & 17,6677 \\
106 & 133 & 327 & 17,6749 & 17,6702 \\
340 & 378 & 427 & 17,6769 & 17,6736 \\
574 & 577 & 606 & 17,6783 & 17,6760 \\
808 & 811 & 914 & 17,6794 & 17,6778 \\
917 & 956 & 981 & 17,6795 & 17,6781 \\
1000 & 1079 & 1090 & 17,6797 & 17,6784 \\
\hline
\end{tabular}

TABLE 4: The influence of the price on the catches.

\begin{tabular}{lcccc}
\hline$p_{01}$ & $p_{02}$ & $p_{03}$ & $H_{1}$ & $H_{2}$ \\
\hline 1 & 2 & 3 & 245,0957 & 234,4651 \\
11 & 17 & 23 & 246,4411 & 245,9382 \\
16 & 27 & 48 & 246,5725 & 246,2429 \\
31 & 47 & 78 & 246,6298 & 246,3781 \\
51 & 70 & 108 & 246,6552 & 246,5718 \\
84 & 101 & 273 & 246,6865 & 246,5974 \\
106 & 133 & 327 & 246,6923 & 246,6334 \\
340 & 378 & 427 & 246,7020 & 246,6334 \\
574 & 577 & 606 & 246,7063 & 246,6582 \\
808 & 811 & 914 & 246,7095 & 246,6775 \\
917 & 956 & 981 & 246,7101 & 246,6804 \\
1000 & 1079 & 1090 & 246,7107 & 246,6839 \\
\hline
\end{tabular}

4.1. The Influence of the Price on the Fishing Effort, Catches, and Profit. By Tables 3, 4, and 5 we will discover how changes in the price can affect the fishing effort, catches, and profit.

According to Tables 3, 4, and 5, one can remark that an increase in the price leads to an increase in fishing effort, catches, and profit. But it is clear that when the price 
TABLE 5: The influence of the price on the profits.

\begin{tabular}{lcccc}
\hline$p_{01}$ & $p_{02}$ & $p_{03}$ & $\pi_{1}$ & $\pi_{2}$ \\
\hline 1 & 2 & 3 & 282 & 269 \\
11 & 17 & 23 & 2959 & 2942 \\
16 & 27 & 48 & 4513 & 4500 \\
31 & 47 & 78 & 8479 & 8465 \\
51 & 70 & 108 & 13584 & 13567 \\
84 & 101 & 273 & 23130 & 23118 \\
106 & 133 & 327 & 29111 & 29099 \\
340 & 378 & 427 & 85598 & 85572 \\
574 & 577 & 606 & 142017 & 141987 \\
808 & 811 & 914 & 200558 & 200531 \\
917 & 956 & 981 & 227721 & 227692 \\
1000 & 1079 & 1090 & 249295 & 249266 \\
\hline
\end{tabular}

level increases significantly, that is, when it varies in a large amplitude interval, the fishing effort and the catches increase by varying in an interval of small amplitude. More precisely, when the price is between 1 and 1090, the fishing effort varies between 16,51 and 17,68, and the catches vary between 234,4 and 246,7 . This is justified by the need for conservation of marine species even if the price increases significantly.

From Table 5 one can see that the level of profit increases, which allows fishermen to have highest returns through more reasonable catches, taking into account the conservation of biodiversity.

These results allow us to deduce that our model is pertinent since it allows us to determine the fishing effort that maximizes the profit of each fisherman without being obliged to make more catches that lead to the overexploitation of these marine species.

Let us add that when the price tends to infinity, the fishing efforts of the two fishermen are equal and they do not exceed 18, as well as the catches which do not exceed 250; contrariwise the profit is always increasing thanks to the increase of the price. Then we can deduce the effect of the price change on the fishing effort, catches, and profit.

It is very interesting to note that if the price tends to infinity and the fishing effort is superior to 18 , then the catches and the profit decrease.

\section{Conclusions}

In this paper, we have developed a bioeconomic model for three species catches by two fishermen. In one hand, we have assumed that the evolution of these species is described by a density dependent model taking into account the competition between the species which compete with each other for space or food. The natural growth of each species is modeled using a logistic law. On the other hand, we have assumed that the prices of these species vary according to the quantity harvested. In this work we have calculated fishing effort that maximizes the income of each fisherman at biological equilibrium by using the Nash equilibrium problem. The existence of the steady states and their stability are studied using eigenvalue analysis and Routh-Hurwitz criterion.

\section{Conflicts of Interest}

The authors declare that there are no conflicts of interest.

\section{References}

[1] C. W. Clark and G. R. Munro, "The economics of fishing and modern capital theory: a simplified approach," Journal of Environmental Economics and Management, vol. 2, no. 2, pp. 92106, 1975.

[2] C. W. Clark, Bioeconomic modelling and fisheries management, John Wiley and Sons, New York, NY, USA, 1985.

[3] K. S. Chaudhuri, "A bioeconomic model of harvesting a multispecies fishery," Ecological Modelling, vol. 32, no. 4, pp. 267-279, 1986.

[4] K. Chaudhuri, "Dynamic optimization of combined harvesting of a two-species fishery," Ecological Modelling, vol. 41, no. 1-2, pp. 17-25, 1988.

[5] K. S. Chaudhuri and S. S. Ray, "On the combined harvesting of a prey-predator system," Journal of Biological Systems, vol. 4, no. 3, pp. 373-389, 1996.

[6] T. K. Kar and K. S. Chaudhuri, "Harvesting in a two-prey onepredator fishery: A bioeconomic model," Anziam Journal, vol. 45, no. 3, pp. 443-456, 2004.

[7] R. Mchich, P. M. Auger, R. Bravo De La Parra, and N. Raissi, "Dynamics of a fishery on two fishing zones with fish stock dependent migrations: Aggregation and control," Ecological Modelling, vol. 158, no. 1-2, pp. 51-62, 2002.

[8] Y. El Foutayeni, M. Khaladi, and A. Zegzouti, Profit maximization of fishermen exploiting two fish species in competition, vol. 15 of AMO Advanced Modeling and Optimisation, 2013, 457-469.

[9] Y. El Foutayeni, M. Khaladi, and A. Zegzouti, A generalized Nash equilibrium for a bioeconomic porblem of fishing, Studia Informatica Universalis-HERMANN, 2012, 186-204.

[10] Y. EL Foutayeni and M. Khaladi, "A bio-economic model of fishery where prices depend on harvest," Advanced Modeling and Optimization. The Electronic International Journal, vol. 14, no. 3, pp. 543-555, 2012.

[11] Y. El Foutayeni and M. Khaladi, "A generalized bio-economic model for competing multiple-fish populations where prices depend on harvest," Advanced Modeling and Optimization. The Electronic International Journal, vol. 14, no. 3, pp. 531-542, 2012.

[12] Mailleret Ludovic, Stabilisation Globale de Systèmes Dynamiques Positifs Mal Connus, Applications en Biologie. Ecologie, Environnement, Université Nice Sophia Antipolis, 2004.

[13] G. Birkhoff and G.-C. Rota, Ordinary Differential Equations, Ginn, 1982. 


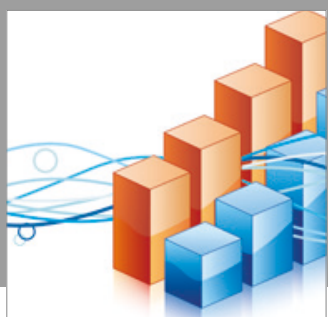

Advances in

Operations Research

vatersals

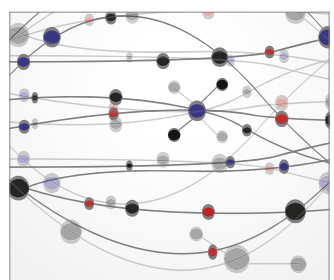

\section{The Scientific} World Journal
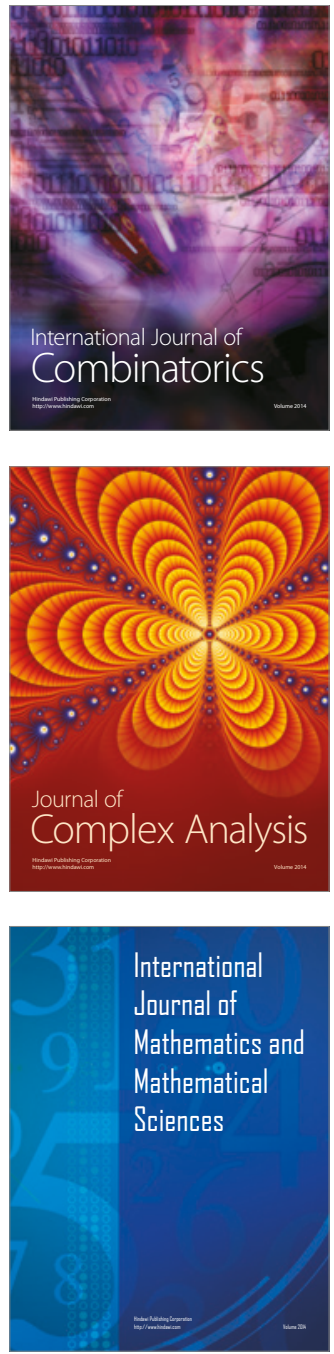
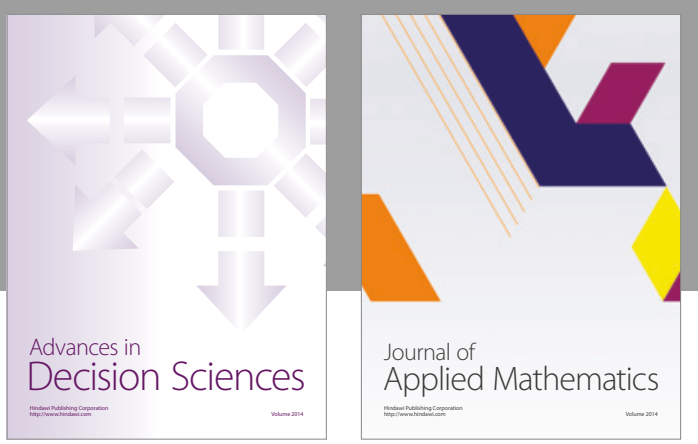

Algebra

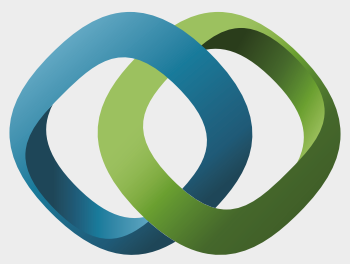

\section{Hindawi}

Submit your manuscripts at

https://www.hindawi.com
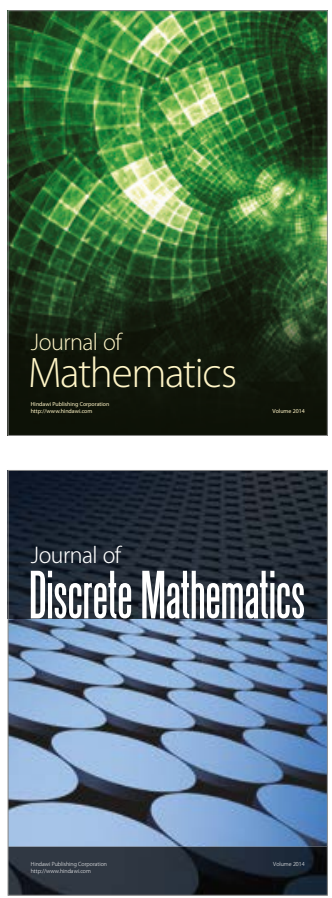

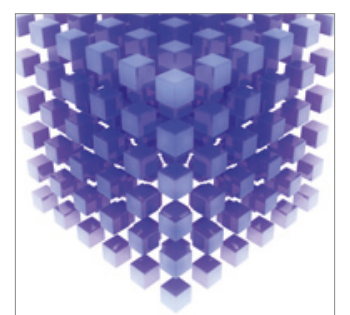

Mathematical Problems in Engineering
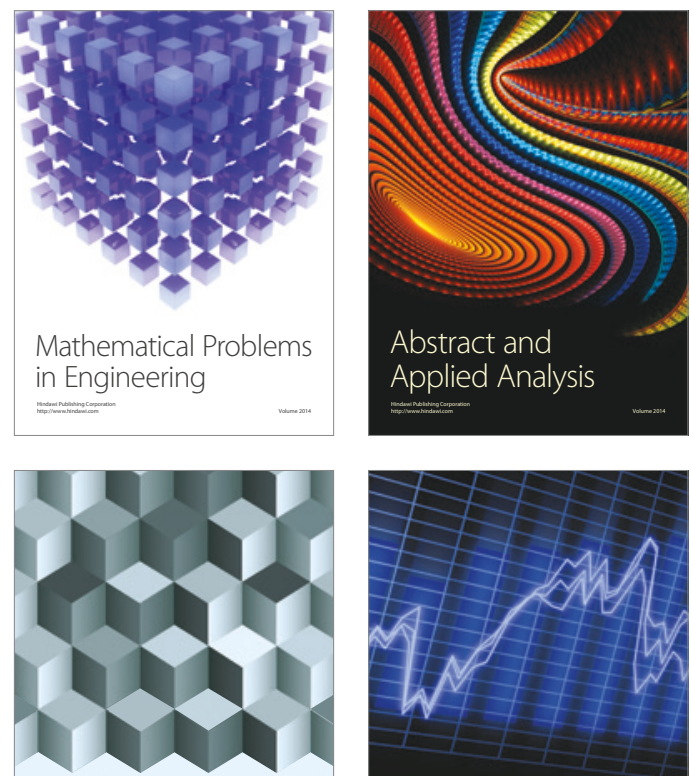

Journal of

Function Spaces

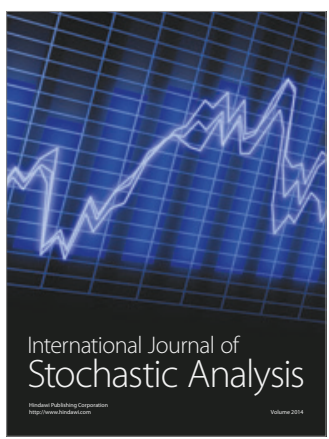

Probability and Statistics
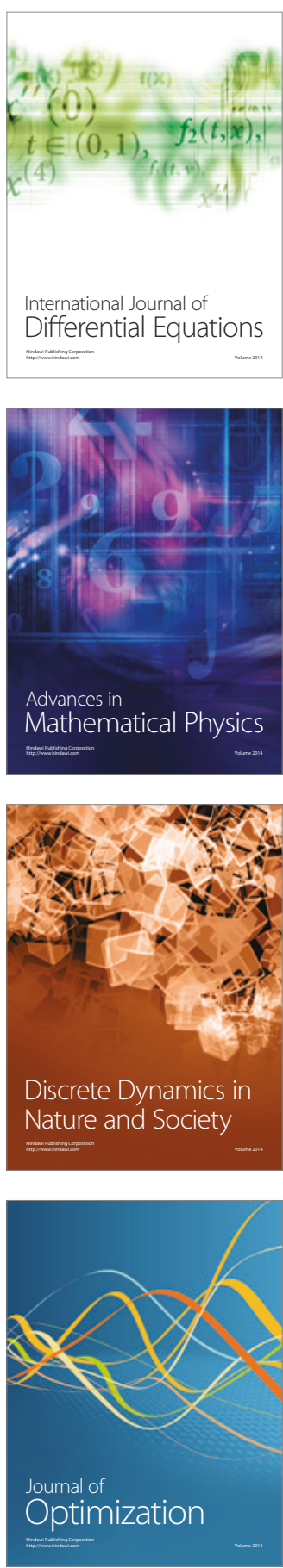